Pacific

Journal of

Mathematics

\title{
MULTIPLICATIVE REDUCTION \\ AND THE CYCLOTOMIC MAIN CONJECTURE FOR GL2
}

CHRISTOPHER SKINNER 


\title{
MULTIPLICATIVE REDUCTION AND THE CYCLOTOMIC MAIN CONJECTURE FOR GL
}

\author{
CHRISTOPHER SKINNER
}

\begin{abstract}
We show that the cyclotomic Iwasawa-Greenberg main conjecture holds for a large class of modular forms with multiplicative reduction at $p$, extending previous results for the good ordinary case. In fact, the multiplicative case is deduced from the good case through the use of Hida families and a simple Fitting ideal argument.
\end{abstract}

\section{Introduction}

The cyclotomic Iwasawa-Greenberg main conjecture was established in [Skinner and Urban 2014], in combination with work of Kato [2004], for a large class of newforms $f \in S_{k}\left(\Gamma_{0}(N)\right)$ that are ordinary at an odd prime $p \nmid N$, subject to $k \equiv 2(\bmod p-1)$ and certain conditions on the $\bmod p$ Galois representation associated with $f$. The purpose of this note is to extend this result to the case where $p \mid N$ (in which case $k$ is necessarily equal to 2).

Recall that the coefficients $a_{n}$ of the $q$-expansion $f=\sum_{n=1}^{\infty} a_{n} q^{n}$ of $f$ at the cusp at infinity (equivalently, the Hecke eigenvalues of $f$ ) are algebraic integers that generate a finite extension $\mathbb{Q}(f) \subset \mathbb{C}$ of $\mathbb{Q}$. Let $p$ be an odd prime and let $L$ be a finite extension of the completion of $\mathbb{Q}(f)$ at a chosen prime above $p$ (equivalently, let $L$ be a finite extension of $\mathbb{Q}_{p}$ in a fixed algebraic closure $\overline{\mathbb{Q}}_{p}$ of $\mathbb{Q}_{p}$ that contains the image of a chosen embedding $\left.\mathbb{Q}(f) \hookrightarrow \overline{\mathbb{Q}}_{p}\right)$. Suppose that $f$ is ordinary at $p$ with respect to $L$ in the sense that $a_{p}$ is a unit in the ring of integers $\mathcal{O}$ of $L$. Then the $p$-adic $L$-function $\mathcal{L}_{f}$ of $f$ is an element of the Iwasawa algebra $\Lambda_{\mathcal{O}}=\mathcal{O} \llbracket \Gamma \rrbracket$, where $\Gamma=\operatorname{Gal}\left(\mathbb{Q}_{\infty} / \mathbb{Q}\right)$ is the Galois group of the cyclotomic $\mathbb{Z}_{p}$-extension $\mathbb{Q}_{\infty}$ of $\mathbb{Q}$. A defining property of $\mathcal{L}_{f}$ is that it interpolates normalized special values of the $L$-function of $f$ twisted by Dirichlet characters associated with finite-order characters of $\Gamma$. The Iwasawa-Greenberg Selmer group $\operatorname{Sel}_{\mathbb{Q}_{\infty}, L}(f)$, defined with respect to the $p$-adic Galois representation $V_{f}$ of $f$ over $L-$ a two-dimensional $L$-vector space - and a Galois-stable $\mathcal{O}$-lattice $T_{f} \subset V_{f}$, is a discrete, cofinite $\Lambda_{\mathcal{O}}$-module, and the Iwasawa-Greenberg characteristic ideal $\mathrm{Ch}_{L}(f) \subset \Lambda_{\mathcal{O}}$ is the characteristic $\Lambda_{\mathcal{O}}$-ideal of the Pontryagin dual $X_{\mathbb{Q}_{\infty}, L}(f)$ of $\operatorname{Sel}_{\mathbb{Q}_{\infty}, L}(f)$. The

MSC2010: primary 11G40, 11R23; secondary 11F67.

Keywords: Iwasawa theory, special values of L-functions, Selmer groups. 
Iwasawa-Greenberg main conjecture for $f$ then asserts that there is an equality of ideals $\mathrm{Ch}_{L}(f)=\left(\mathcal{L}_{f}\right)$ in $\Lambda_{\mathcal{O}} \otimes_{\mathbb{Z}_{p}} \mathbb{Q}_{p}$ and even in $\Lambda_{\mathcal{O}}$ if $T_{f}$ is residually irreducible. Theorem A. Let $p \geq 3$ be a prime. Let $f \in S_{k}\left(\Gamma_{0}(N)\right)$ be a newform and let $L$ and $\mathcal{O}$ be as above and suppose $f$ is ordinary at $p$ with respect to $L$. If

(i) $k \equiv 2(\bmod p-1)$;

(ii) the reduction $\bar{\rho}_{f}$ of the representation $\rho_{f}: \mathrm{Gal}(\overline{\mathbb{Q}} / \mathbb{Q}) \rightarrow \operatorname{Aut}_{\mathcal{O}}\left(T_{f}\right)$ modulo the maximal ideal of $\mathcal{O}$ is irreducible;

(iii) there exists a prime $q \neq p$ such that $q \| N$ and $\bar{\rho}_{f}$ is ramified at $q$, then $\mathrm{Ch}_{L}(f)=\left(\mathcal{L}_{f}\right)$ in $\Lambda_{\mathcal{O}}$. That is, the Iwasawa-Greenberg main conjecture is true.

When $p \nmid N$ this is just Theorem 1 of [Skinner and Urban 2014] ${ }^{1}$. When $p \mid N$, in which case the ordinary hypothesis forces $p \| N$ and $k=2$, this is not an immediate consequence of the results in [Skinner and Urban 2014], as this case is excluded from Kato's divisibility theorem [2004, Theorem 17.4], which is a crucial ingredient in the deduction of the main conjecture from the main results in [Skinner and Urban 2014]. However, as we explain in this note, the main conjecture in the case $p \mid N$ can be deduced from knowing it when $p \nmid N$.

Having the cyclotomic main conjecture in hand, one obtains results toward special value formulas. For example:

Theorem B. Let $p \geq 3$ be a prime. Let $f \in S_{2}\left(\Gamma_{0}(N)\right)$ be a newform and let $L$ and $\mathcal{O}$ be as above and suppose $f$ is ordinary. Suppose also that

(i) the reduction $\bar{\rho}_{f}$ of the representation $\rho_{f}: \operatorname{Gal}(\overline{\mathbb{Q}} / \mathbb{Q}) \rightarrow \operatorname{Aut}_{\mathcal{O}}\left(T_{f}\right)$ modulo the maximal ideal of $\mathcal{O}$ is irreducible;

(ii) there exists a prime $q \neq p$ such that $q \| N$ and $\bar{\rho}_{f}$ is ramified at $q$;

(iii) if $p \mid N$ and $a_{p}=1$, then the $\mathfrak{L}$-invariant $\mathfrak{L}\left(V_{f}\right) \in L$ is nonzero.

Let

$$
L^{\mathrm{alg}}(f, 1)=\frac{L(f, 1)}{-2 \pi i \Omega_{f}^{+}}
$$

Then

$$
\# \mathcal{O} /\left(L^{\operatorname{alg}}(f, 1)\right)=\# \operatorname{Sel}_{L}(f) \cdot \prod_{\ell} c_{\ell}\left(T_{f}\right) .
$$

In particular, if $L(f, 1)=0$, then $\operatorname{Sel}_{L}(f)$ has $\mathcal{O}$-corank at least one.

\footnotetext{
${ }^{1}$ In order to conclude that the equality holds in $\Lambda_{\mathcal{O}}$ and not just $\Lambda_{\mathcal{O}} \otimes_{\mathbb{Z}_{p}} \mathbb{Q}_{p}$, Theorem 1 in [Skinner and Urban 2014] requires that $\rho_{f}$ have an $\mathcal{O}$-basis with respect to which the image contains $\mathrm{SL}_{2}\left(\mathbb{Z}_{p}\right)$. But as we explain in Section 2.5, hypotheses (ii) and (iii) of Theorem A are enough for the arguments. We also explain that the reference to [Vatsal 2003] in [Skinner and Urban 2014] should have been augmented with a reference to [Chida and Hsieh 2016].
} 
Here $\Omega_{f}^{+}$is one of two canonical periods associated with $f$ as in [Skinner and Urban 2014, §3.3.3] (and well-defined up to an element of $\mathcal{O}^{\times} \cap \mathbb{Q}(f)$ ), $\operatorname{Sel}_{L}(f)$ is the Selmer group associated by Bloch-Kato to the Galois lattice $T_{f}, c_{\ell}\left(T_{f}\right)$ is the Tamagawa factor at $\ell$ of $T_{f}$ (and equals 1 unless $\ell \mid N$ ), and $\mathfrak{L}\left(V_{f}\right)$ is the $\mathcal{L}$-invariant of a modular form $f$ (or of $V_{f}$ ) with split multiplicative reduction at $p$ introduced by Mazur, Tate, and Teitelbaum [1986] (see also [Greenberg and Stevens 1993, §3]). It is conjectured that $\mathfrak{L}\left(V_{f}\right)$ is always nonzero; this is known if $f$ is the modular form associated to an elliptic curve, but in general it is an open question.

As a special case of Theorem $\mathrm{B}$, obtained by taking $f$ to be the newform associated with an elliptic curve $E$ over $\mathbb{Q}$, we have:

Theorem $\mathbf{C}$. Let $E$ be an elliptic curve over $\mathbb{Q}$ with good ordinary or multiplicative reduction at a prime $p \geq 3$. Suppose that

(i) $E[p]$ is an irreducible $\mathrm{Gal}(\overline{\mathbb{Q}} / \mathbb{Q})$-representation;

(ii) there exists a prime $q \neq p$ at which $E$ has multiplicative reduction and $E[p]$ is ramified.

If $L(E, 1) \neq 0$ then

$$
\operatorname{ord}_{p}\left(\frac{L(E, 1)}{\Omega_{E}}\right)=\operatorname{ord}_{p}\left(\# \amalg(E) \prod_{\ell} c_{\ell}(E)\right),
$$

and if $L(E, 1)=0$ then $\operatorname{Sel}_{p}(E)$ has $\mathbb{Z}_{p}$-corank at least one.

Here, $\Omega_{E}$ is the Néron period of $E, \amalg(E)$ is the Tate-Shafarevich group of $E / \mathbb{Q}$, and the $c_{\ell}(E)$ are the Tamagawa numbers of $E$. In particular, $c_{\ell}(E)$ is the order of the group of irreducible components of the special fiber of the Néron model of $E$ over $\mathbb{Z}_{\ell}$.

Our proof of Theorem A is relatively simple. Let $N=p M$. We first make two reductions: (1) it suffices to prove the theorem with the field $L$ replaced by any finite extension, and (2) it suffices to prove the equality $\operatorname{Ch}_{L}^{\Sigma}(f)=\left(\mathcal{L}_{f}^{\Sigma}\right)$, where $\Sigma$ is any finite set of primes containing all $\ell \mid N, \mathcal{L}_{f}^{\Sigma}$ is the incomplete $p$-adic $L$-function with the Euler factors at primes in $\Sigma$ different from $p$ removed, and $\mathrm{Ch}_{L}^{\Sigma}(f)$ is the characteristic ideal of the Pontryagin dual $X_{\mathbb{Q}_{\infty}, L}^{\Sigma}(f)$ of the Iwasawa-Greenberg Selmer group $\operatorname{Sel}_{\mathbb{Q}_{\infty}, L}^{\Sigma}(f)$ with all conditions at primes in $\Sigma$ different from $p$ relaxed. Then we exploit Hida theory to deduce that one can choose $L$ so that for each integer $m>0$ there exists a newform $f_{m} \in S_{k_{m}}\left(\Gamma_{0}(M)\right)$ with $k_{m} \equiv k\left(\bmod (p-1) p^{m}\right), \mathbb{Q}\left(f_{m}\right) \subset L$ and $f_{m}$ ordinary at $p$ with respect to $L$, and the ordinary $p$-stabilization $f_{m}^{*}$ of $f_{m}$ satisfies $f_{m}^{*} \equiv f\left(\bmod p^{m}\right)$ in the sense that the $q$-expansions (which have coefficients in $\mathcal{O}$ ) are congruent modulo $p^{m}$. Furthermore, as a consequence of the existence of the "two-variable" $p$-adic $L$-function associated to a Hida family we also have $\mathcal{L}_{f_{m}}^{\Sigma} \equiv \mathcal{L}_{f}^{\Sigma}\left(\bmod p^{m} \Lambda_{\mathcal{O}}\right)$. Kato 
[2004] has proved that $X_{\mathbb{Q}_{\infty}, L}^{\Sigma}\left(f_{m}\right)$ is a torsion $\Lambda_{\mathcal{O}}$-module, and an argument of Greenberg then shows that it has no nonzero finite-order $\Lambda_{\mathcal{O}}$-submodules. From this it follows that $\mathrm{Ch}_{L}^{\Sigma}\left(f_{m}\right)$ equals the $\Lambda_{\mathcal{O}}$-Fitting ideal $F_{L}^{\Sigma}\left(f_{m}\right)$ of $X_{L}^{\Sigma}\left(f_{m}\right)$. The congruence $f_{m}^{*} \equiv f\left(\bmod p^{m}\right)$ implies that $\operatorname{Sel}_{\mathbb{Q}_{\infty}, L}^{\Sigma}(f)\left[p^{m}\right] \cong \operatorname{Sel}_{\mathbb{Q}_{\infty}, L}^{\Sigma}\left(f_{m}\right)\left[p^{m}\right]$, so comparing Fitting ideals yields

$$
\left(F_{L}^{\Sigma}(f), p^{m}\right)=\left(F_{L}^{\Sigma}\left(f_{m}\right), p^{m}\right)=\left(\mathrm{Ch}_{L}^{\Sigma}\left(f_{m}\right), p^{m}\right) \subset \Lambda_{\mathcal{O}} .
$$

From the main conjecture for $f_{m}$ (the congruence $f_{m}^{*} \equiv f(\bmod p)$ ensures that the hypotheses of Theorem A also hold for $f_{m}$ ) and the congruence modulo $p^{m}$ of $p$-adic $L$-functions we then have

$$
\left(F_{L}^{\Sigma}(f), p^{m}\right)=\left(\mathrm{Ch}_{L}^{\Sigma}\left(f_{m}\right), p^{m}\right)=\left(\mathcal{L}_{f_{m}}^{\Sigma}, p^{m}\right)=\left(\mathcal{L}_{f}^{\Sigma}, p^{m}\right) \subset \Lambda_{\mathcal{O}}
$$

for all integers $m>0$. This, together with the nonvanishing of the $p$-adic $L$ function $\mathcal{L}_{f}^{\Sigma}$, implies that $F_{L}^{\Sigma}(f) \neq 0$ and hence that $X_{\mathbb{Q}_{\infty}, L}^{\Sigma}(f)$ is a torsion $\Lambda_{\mathcal{O}^{-}}$ module. Then $\mathrm{Ch}_{L}^{\Sigma}(f)=F_{L}^{\Sigma}(f)$, by the earlier argument of Greenberg, and so $\left(\mathrm{Ch}_{L}^{\Sigma}(f), p^{m}\right)=\left(\mathcal{L}^{\Sigma}(f), p^{m}\right) \subset \Lambda_{\mathcal{O}}$ for all $m>0 . \mathrm{As}^{\mathrm{Ch}_{L}^{\Sigma}}(f) \subset \Lambda_{\mathcal{O}}$ is a principal ideal, it then easily follows that $\operatorname{Ch}_{L}^{\Sigma}(f)=\left(\mathcal{L}_{f}^{\Sigma}\right)$, proving Theorem A.

If the analytic or algebraic $\mu$-invariant for some $f_{m}$ (the power of the uniformizer of $L$ dividing $\mathcal{L}_{f_{m}}^{\Sigma}$ or $\left.\mathrm{Ch}_{L}^{\Sigma}\left(f_{m}\right)\right)$ - or even for some other ordinary eigenform suitably congruent to $f$ for which the main conjecture holds - were known to be zero, then Theorem A would follow from the main results of [Emerton et al. 2006]. However, presently little is known about the vanishing of these $\mu$-invariants.

Theorem B is deduced from Theorem A via an argument of Greenberg [1999].

In addition to extending the main conjecture to the case of multiplicative reduction, our motivation for writing this note was in part to provide an explicit reference for the expression for the special value $L^{\text {alg }}(f, 1)$ in terms of the size of Selmer groups that is required for the arguments in [Zhang 2014] and, by including the multiplicative reduction case, also to provide an important ingredient for the extension of the main results of [Zhang 2014] to cases of multiplicative reduction. Additional motivation for the latter stems from the author's collaboration with Manjul Bhargava and Wei Zhang to provide lower bounds on the proportion of elliptic curves that satisfy the rank part of the Birch-Swinnerton-Dyer conjecture.

While preparing this note the author learned of Olivier Fouquet's [2014] work on the equivariant Tamagawa number conjecture for motives of modular forms. That work should provide another means for deducing Theorem B in the case $p \mid N$ from the main results ${ }^{2}$ in [Skinner and Urban 2014] as well as some additional weakening of the conditions on primes away from $p$. The deduction of Theorem A

${ }^{2}$ But see also note 1, especially as the main results in [Fouquet 2014] rely on Theorem A as stated, at least for the $p \nmid N$ case. 
for $p \mid N$ in this paper uses no more machinery than already developed in [Skinner and Urban 2014] or than is required for our deduction of Theorem B.

\section{Gathering the pieces}

In this section we recall the various objects that go into the Iwasawa-Greenberg main conjecture for modular forms, some of their properties, and some useful relations. Throughout $p$ is a fixed odd prime.

Let $\overline{\mathbb{Q}} \subset \mathbb{C}$ be the algebraic closure of $\mathbb{Q}$ and let $G_{\mathbb{Q}}=\operatorname{Gal}(\overline{\mathbb{Q}} / \mathbb{Q})$. For each prime $\ell$, let $\overline{\mathbb{Q}}_{\ell}$ be a fixed algebraic closure of $\overline{\mathbb{Q}}_{\ell}$. For each $\ell$ we also fix an embedding $\overline{\mathbb{Q}} \hookrightarrow \overline{\mathbb{Q}}_{\ell}$, which identifies $G_{\mathbb{Q}_{\ell}}=\operatorname{Gal}\left(\overline{\mathbb{Q}}_{\ell} / \overline{\mathbb{Q}}\right)$ with a decomposition subgroup in $G_{\mathbb{Q}_{\mathbb{Q}}}$; let $I_{\ell} \subset G_{\mathbb{Q}_{\ell}}$ be the inertia subgroup. Let frob ${ }_{\ell} \in G_{\mathbb{Q}_{\ell}}$ be (a lift of) an arithmetic Frobenius element.

Let $\epsilon: G_{\mathbb{Q}} \rightarrow \mathbb{Z}_{p}^{\times}$be the $p$-adic cyclotomic character. This is just the projection to $\operatorname{Gal}\left(\mathbb{Q}\left[\mu_{p^{\infty}}\right] / \mathbb{Q}\right)$, the latter being canonically isomorphic to $\mathbb{Z}_{p}^{\times}$. Similarly, let $\omega: G_{\mathbb{Q}} \rightarrow \mathbb{Z}_{p}^{\times}$be the mod $p$ Teichmüller character. This is just the composition of the reduction of $\epsilon \bmod p$ and the multiplicative homomorphism $(\mathbb{Z} / p \mathbb{Z})^{\times} \hookrightarrow \mathbb{Z}_{p}^{\times}$ defined by the Teichmüller lifts.

Let $\mathbb{Q}_{\infty} \subset \mathbb{Q}\left[\mu^{p \infty}\right] \subset \overline{\mathbb{Q}}$ be the cyclotomic $\mathbb{Z}_{p}$-extension of $\mathbb{Q}$. That is, $\mathbb{Q}_{\infty}$ is the unique abelian extension of $\mathbb{Q}$ such that $\Gamma=\operatorname{Gal}\left(\mathbb{Q}_{\infty} / \mathbb{Q}\right) \cong \mathbb{Z}_{p}$. Let $\gamma \in \Gamma$ be a fixed topological generator. As $\operatorname{Gal}\left(\mathbb{Q}\left[\mu_{p^{\infty}}\right] / \mathbb{Q}\right) \stackrel{\sim}{\rightarrow} \operatorname{Gal}\left(\mathbb{Q}\left[\mu_{p}\right] / \mathbb{Q}\right) \times \Gamma$, there is a lift $\tilde{\gamma}$ of $\gamma$ to $\operatorname{Gal}\left(\mathbb{Q}\left[\mu_{p^{\infty}}\right] / \mathbb{Q}\right)$ identified with $(1, \gamma)$, and we let $u=\epsilon(\tilde{\gamma}) \in \mathbb{Z}_{p}^{\times}$.

2.1. Galois representations and (ordinary) newforms. Let $f \in S_{k}\left(\Gamma_{0}(N)\right)$ be a newform. Let $\mathbb{Q}(f) \subset \mathbb{C}$ be the finite extension of $\mathbb{Q}$ generated by the Fourier coefficients $a_{n}(f)$ of the $q$-expansion $f=\sum_{n=0}^{\infty} a_{n}(f) q^{n}$ of $f$ at the cusp at infinity (equivalently, the field obtained by adjoining the eigenvalues of the action of the usual Hecke operators on $f$ ). Fix an embedding $\mathbb{Q}(f) \hookrightarrow \overline{\mathbb{Q}}_{p}$ and let $L \subset \overline{\mathbb{Q}}_{p}$ be a finite extension of $\mathbb{Q}_{p}$ containing the image of $\mathbb{Q}(f)$. Let $\mathcal{O}$ be the ring of integers of $L$ (the valuation ring), let $\mathfrak{m}$ be its maximal ideal, and let $\kappa=\mathcal{O} / \mathfrak{m}$ be its residue field.

Associated with $f$ and $L$ (and the embedding $\mathbb{Q}(f) \hookrightarrow L)$ is a two-dimensional $L$-space $V_{f}$ and an absolutely irreducible continuous $G_{\mathbb{Q}}$-representation $\rho_{f}: G_{\mathbb{Q}} \rightarrow$ $\operatorname{Aut}_{L}\left(V_{f}\right)$ such that $\rho_{f}$ is unramified at all primes $\ell \nmid N p$ and $\operatorname{det}\left(1-X \cdot \rho_{f}\left(\operatorname{frob}_{\ell}\right)\right)=$ $1-a_{\ell}(f) X+\ell^{k-1} X^{2}$ for such $\ell$. In particular, trace $\rho_{f}\left(\right.$ frob $\left._{\ell}\right)=a_{\ell}(f)$ if $\ell \nmid p N$, and $\operatorname{det} \rho_{f}=\epsilon^{k-1}$.

Let $T, T^{\prime} \subset V_{f}$ be two $G_{\mathbb{Q}^{-}}$-stable $\mathcal{O}$-lattices. Let $\bar{\rho}$ and $\bar{\rho}^{\prime}$ denote, respectively, the two-dimensional $\kappa$-representations $T / \mathfrak{m} T$ and $T^{\prime} / \mathfrak{m} T^{\prime}$. The following lemma is well known, but we include it for later reference.

Lemma 2.1.1. (a) If $\bar{\rho}$ or $\bar{\rho}^{\prime}$ is irreducible, then $\bar{\rho}$ and $\bar{\rho}^{\prime}$ are equivalent as $\kappa$ representations. In particular, $\bar{\rho}$ is irreducible if and only if $\bar{\rho}^{\prime}$ is irreducible. 
(b) If $\bar{\rho}$ or $\bar{\rho}^{\prime}$ is irreducible, then there exists $a \in L^{\times}$such that $T=a T^{\prime}$.

Proof. Replacing $T^{\prime}$ with some $\mathcal{O}$-multiple, we may assume that $T^{\prime}$ is a sublattice of $T$. Then $T / T^{\prime} \cong \mathcal{O} / \mathfrak{m}^{n} \times \mathcal{O} / \mathfrak{m}^{m}$ with $n \leq m$. Let $\varpi$ be a uniformizer of $\mathcal{O}$ (a generator of $\mathfrak{m})$. Then $\varpi^{n} T /\left(T^{\prime}+\varpi^{n+1} T\right) \cong \mathcal{O} / \mathfrak{m}^{\min (1, m-n)}$ is a $G_{\mathbb{Q}}$-stable quotient of $T / \mathfrak{m} T \cong \varpi^{n} T / \varpi^{n+1} T$ of at most one-dimension over $k$. If $\bar{\rho}$ is irreducible, then this quotient must be trivial and so $m-n=0$ and $T^{\prime}=\varpi^{n} T$, in which case $T^{\prime} / \mathfrak{m} T^{\prime} \cong \varpi^{n} T / \varpi^{n+1} T \cong T / \mathfrak{m} T$ as $G_{\mathbb{Q}}$-representations over $\kappa$. Reversing the roles of $T$ and $T^{\prime}$ in this argument then yields the lemma.

We then define $\bar{\rho}_{f}$ to be the $\kappa$-representation $T / \mathfrak{m} T$ of $G_{\mathbb{Q}}$ for a Galois-stable $\mathcal{O}$-lattice $T \subset V_{f}$. By the above lemma, if $\bar{\rho}_{f}$ is irreducible for some choice of $T$, then it is irreducible for any choice of $T$, and the equivalence class of $\bar{\rho}_{f}$ is independent of $T$. Of course, it is not difficult to show that the semisimplification of $\bar{\rho}_{f}$ is independent of $T$ even when $\bar{\rho}_{f}$ is not irreducible, but will not need this.

Suppose $k \geq 2$ and $f$ is ordinary with respect to the embedding $\mathbb{Q}(f) \hookrightarrow L$. That is, $a_{p}(f) \in \mathcal{O}^{\times}$. As proved in general by Wiles [1988, Theorem 2.2.2], in this case $V_{f}$ has a unique $G_{\mathbb{Q}_{p}}$-stable $L$-line $V_{f}^{+} \subset V_{f}$ such that $G_{\mathbb{Q}_{p}}$ acts on $V_{f}^{+}$via the character $\alpha_{f}^{-1} \epsilon^{k-1}$, where $\alpha_{f}: G_{\mathbb{Q}_{p}} \rightarrow \mathcal{O}^{\times}$is the unique unramified character such that $\alpha_{f}\left(\operatorname{frob}_{p}\right.$ ) equals the (unit) root $\alpha_{p}$ in $\mathcal{O}^{\times}$of the polynomial $x^{2}-a_{p}(f) x+p^{k-1}$ if $p \nmid N$ and $\alpha_{f}\left(\right.$ frob $\left._{p}\right)=a_{p}(f)$ if $p \mid N$. (Note that the reduction of the polynomial $x^{2}-a_{p}(f) x+p^{k-1}$ modulo $\mathfrak{m}$ is $x\left(x-\bar{a}_{p}(f)\right)$ and so, by Hensel's lemma, $\bar{a}_{p}(f)$ lifts to a root in $\mathcal{O}^{\times}$.) The action of $G_{\mathbb{Q}_{p}}$ on the quotient $V_{f}^{-}=V_{f} / V_{f}^{+}$is via $\alpha_{f}$. Given any $G_{\mathbb{Q}^{-}}$-stable $\mathcal{O}$-lattice $T \subset V_{f}$ we let $T^{+}=T \cap V_{f}^{+}$and $T^{-}=T / T^{+}$. Then $T^{+}$is the unique $G_{\mathbb{Q}_{p}}$-stable free $\mathcal{O}$-summand of rank one on which $G_{\mathbb{Q}_{p}}$ acts via $\alpha_{f}^{-1} \epsilon^{k-1}$, and $T^{-}$is the unique $G_{\mathbb{Q}_{p}}$-stable free $\mathcal{O}$-module quotient of rank one on which $G_{\mathbb{Q}_{p}}$ acts via $\alpha_{f}$.

The following lemma is also well known, but we also include it for completeness.

Lemma 2.1.2. Suppose $a_{p}(f) \in \mathcal{O}^{\times}$. If $p \mid N$, then $p \| N, k=2$, and $a_{p}(f)= \pm 1$.

Proof. If $f \in S_{k}\left(\Gamma_{0}(N)\right)$ is a newform with trivial Nebentypus such that $p \mid N$, then $a_{p}(f) \neq 0$ if and only if $p \| N$, in which case $a_{p}(f)^{2}=p^{k-2}$ (see [Miyake 1989, Theorem 4.6.17]). If $a_{p}(f) \in \mathcal{O}^{\times}$, then it follows that $k=2$ and $a_{p}(f)^{2}=1$, so $a_{p}(f)= \pm 1$.

Note that if $f$ is a newform with $p \mid N$ that is ordinary with respect to some embedding $\mathbb{Q}(f) \hookrightarrow \overline{\mathbb{Q}}_{p}$, then, since $a_{p}(f)= \pm 1$ by the lemma, it is ordinary with respect to all such embeddings. Also, as noted in the proof of the lemma, if $f \in S_{2}\left(\Gamma_{0}(N)\right)$ is a newform with $p \| N$ then $a_{p}(f)= \pm 1$ and so $f$ is ordinary with respect to any embedding $\mathbb{Q}(f) \hookrightarrow \overline{\mathbb{Q}}_{p}$.

In keeping with the terminology for elliptic curves, we say that a newform $f \in$ $S_{2}\left(\Gamma_{0}(N)\right)$ has multiplicative reduction at $p$ if $p \| N$ and that it has good reduction 
at $p$ if $p \nmid N$. Additionally, we say $f$ has split (resp. nonsplit) multiplicative reduction at $p$ if $p \| N$ and $a_{p}(f)=1$ (resp. $a_{p}=-1$ ).

2.2. L-invariants. Suppose $f \in S_{2}\left(\Gamma_{0}(N)\right)$ is a newform with split multiplicative reduction at $p$. The Galois representation $V_{f}$ restricted to $G_{\mathbb{Q}_{p}}$ is an extension

$$
0 \rightarrow V_{f}^{+} \cong L(1) \rightarrow V_{f} \rightarrow V_{f}^{-} \cong L \rightarrow 0 .
$$

This extension is known to be nonsplit and semistable but not crystalline. This follows for example from the main result ${ }^{3}$ of [Saito 1997]. Let $\pi_{V_{f}}: H^{1}\left(\mathbb{Q}_{p}, V_{f}\right) \rightarrow$ $H^{1}\left(\mathbb{Q}_{p}, L\right)$ be the induced map on cohomology. As the extension is nonsplit, the image of $\pi_{V_{f}}$ is a one-dimensional $L$-space. As explained in [Greenberg and Stevens 1993, §3], the $\mathfrak{L}$-invariant $\mathfrak{L}\left(V_{f}\right)$ of $V_{f}$ is the negative of the "slope" of the line $\operatorname{im}\left(\pi_{V_{f}}\right)$ with respect to a particular basis of the two-dimensional $L$ space $H^{1}\left(\mathbb{Q}_{p}, L\right)$.

We have

$$
H^{1}\left(\mathbb{Q}_{p}, L\right)=\operatorname{Hom}_{\mathrm{cts}}\left(G_{\mathbb{Q}_{p}}, L\right)=\operatorname{Hom}_{\mathrm{cts}}\left(G_{\mathbb{Q}_{p}}^{\mathrm{ab}, p}, L\right),
$$

where $G_{\mathbb{Q}_{p}}^{\mathrm{ab}, p}$ is the maximal abelian pro- $p$ quotient of $G_{\mathbb{Q}_{p}}$. Local class field theory gives an identification ${ }^{4}$

$$
\underset{n}{\lim _{n}} \mathbb{Q}_{p}^{\times} /\left(\mathbb{Q}_{p}^{\times}\right)^{p^{n}} \stackrel{\sim}{\longrightarrow} G^{\mathrm{ab}, p} .
$$

From the decomposition $\mathbb{Q}_{p}^{\times}=p^{\mathbb{Z}} \times \mathbb{Z}_{p}^{\times}$we obtain an $L$-basis $\left\{\psi_{\mathrm{ur}}, \psi_{\mathrm{cyc}}\right\}$ of $H^{1}\left(\mathbb{Q}_{p}, L\right)=\operatorname{Hom}_{\mathrm{cts}}\left(G_{\mathbb{Q}_{p}}^{\mathrm{ab}, p}, L\right)$, with

$$
\psi_{\mathrm{ur}}(p)=1=\left(\log _{p} u\right)^{-1} \cdot \psi_{\mathrm{cyc}}(u) \text { and } \quad \psi_{\mathrm{ur}}(u)=0=\psi_{\mathrm{cyc}}(p) .
$$

Recall that $u=\epsilon(\tilde{\gamma})$ is a topological generator of $1+p \mathbb{Z}_{p}$. The condition that $V_{f}$ is not crystalline is equivalent to $\operatorname{im}\left(\pi_{V_{f}}\right) \not \subset L \cdot \psi_{\text {ur. }}$ Let $0 \neq \lambda \in \operatorname{im}\left(\pi_{V_{f}}\right)$ and write $\lambda=x \cdot \psi_{\text {cyc }}+y \cdot \psi_{\text {ur }}$. Then $x \neq 0$, and the $\mathfrak{L}$-invariant $\mathfrak{L}\left(V_{f}\right)$ of the extension $V_{f}$ is defined to be

$$
\mathfrak{L}\left(V_{f}\right)=-x^{-1} y \in L .
$$

\footnotetext{
${ }^{3}$ In [Saito 1997] it is proved that the Frobenius semisimplification of the Weil-Deligne representation attached by Fontaine to the dual representation $V_{f}^{\vee}$ is just the Weil-Deligne representation attached by the local Langlands correspondence to the $p$-component $\pi_{p}$ of the automorphic representation $\pi=\otimes_{v} \pi_{v}$ of $\mathrm{GL}_{2}(\mathbb{A})$ corresponding to the newform $f$. If $f$ has split (resp. nonsplit) multiplicative reduction at $p$, then another way to state Lemma 2.1.2 is that $\pi_{p}$ is the special representation (resp. the twist of the special representation by the unramified quadratic character). The local Langlands correspondence attaches to a (twist of a) special representation a Weil-Deligne representation with nontrivial monodromy (in particular, one that is not split).

${ }^{4}$ To be precise, we normalize the reciprocity law so that uniformizers are taken to arithmetic Frobenius elements.
} 
This is independent of the choice of $\lambda$.

The nonsplit extension $V_{f}$ also defines a line $\ell_{V_{f}} \in H^{1}\left(\mathbb{Q}_{p}, L(1)\right)$ (the image of the boundary map $L=H^{0}\left(\mathbb{Q}_{p}, L\right) \rightarrow H^{1}\left(\mathbb{Q}_{p}, L(1)\right)$. Under the perfect pairing $\langle\cdot, \cdot\rangle: H^{1}\left(\mathbb{Q}_{p}, L\right) \times H^{1}\left(\mathbb{Q}_{p}, L(1)\right) \rightarrow H^{2}\left(\mathbb{Q}_{p}, L(1)\right)=L$ of Tate local duality, the lines $\operatorname{im}\left(\pi_{V_{f}}\right)$ and $\ell_{V_{f}}$ are mutual annihilators. So $\mathfrak{L}\left(V_{f}\right)$ can also be expressed in terms of $\left\langle\psi_{\mathrm{ur}}, c\right\rangle$ and $\left\langle\psi_{\mathrm{cyc}}, c\right\rangle$ for $0 \neq c \in \ell_{V_{f}}$.

The Kummer isomorphism yields an identification

$$
\left(\lim _{n} \mathbb{Q}_{p}^{\times} /\left(\mathbb{Q}_{p}^{\times}\right)^{p^{n}}\right) \otimes_{\mathbb{Z}_{p}} L \stackrel{\sim}{\longrightarrow} H^{1}\left(\mathbb{Q}_{p}, L(1)\right) .
$$

Then, together with the above identification of $H^{1}\left(\mathbb{Q}_{p}, L\right)$, the pairing $\langle\cdot, \cdot\rangle$ of local Tate duality is identified with the usual $L$-linear pairing

$$
\operatorname{Hom}_{\mathbb{Z}_{p}}\left(\left({\underset{n}{n}}_{\lim _{p}} \mathbb{Q}_{p}^{\times} /\left(\mathbb{Q}_{p}^{\times}\right)^{p^{n}}\right), L\right) \times\left({\underset{n}{\lim }}_{\mathbb{Q}_{p}^{\times}}^{\times} /\left(\mathbb{Q}_{p}^{\times}\right)^{p^{n}}\right) \otimes_{\mathbb{Z}_{p}} L \rightarrow L .
$$

So if $0 \neq c \in \ell_{V_{f}}$, then

$$
\mathfrak{L}\left(V_{f}\right)=\psi_{\text {ur }}(c)^{-1} \psi_{\text {cyc }}(c) .
$$

Let $H_{f}^{1}\left(\mathbb{Q}_{p}, L(1)\right)$ be the local Bloch-Kato Selmer group [Bloch and Kato 1990, (3.7.2)]. Essentially by definition, $H_{f}^{1}\left(\mathbb{Q}_{p}, L(1)\right)$ is the subgroup of $H^{1}\left(\mathbb{Q}_{p}, L(1)\right)$ that classifies crystalline extensions of $L$ by $L(1)$ (see [loc. cit., p. 354]). The condition that $V_{f}$ not be crystalline is therefore equivalent to $\ell_{V_{f}} \not \subset H_{f}^{1}\left(\mathbb{Q}_{p}, L(1)\right)$, and so, as $H_{f}^{1}\left(\mathbb{Q}_{p}, L(1)\right)$ is identified with $\left(\varliminf_{n} \mathbb{Z}_{p}^{\times} /\left(\mathbb{Z}_{p}^{\times}\right)^{p^{n}}\right) \otimes_{\mathbb{Z}_{p}} L$ (see [loc. cit., Example 3.9]),

$$
\psi_{\mathrm{ur}}(c) \neq 0,
$$

which explains why the preceding formula for $\mathfrak{L}\left(V_{f}\right)$ is well defined.

Example. Suppose $f$ is associated with an elliptic curve $E / \mathbb{Q}$ with split multiplicative reduction at $p$ and let $q_{E} \in \mathbb{Q}_{p}^{\times}$be the Tate period of $E$. Then $V_{f}=T_{p} E \otimes_{\mathbb{Z}_{p}} \mathbb{Q}_{p}$ is the $G_{\mathbb{Q}_{p}}$-extension associated to the image of $q_{E}$ in $H^{1}\left(\mathbb{Q}_{p}, \mathbb{Q}_{p}(1)\right)$ under the Kummer map. That is, $\ell_{V_{f}}=\mathbb{Q}_{p} \cdot q_{E} \in\left(\varliminf_{n} \mathbb{Q}_{p}^{\times} /\left(\mathbb{Q}_{p}^{\times}\right)^{p^{n}}\right) \mathbb{\mathbb { Z }}_{p} \mathbb{Q}_{p}$, and so $\mathfrak{L}\left(V_{f}\right)=\log _{p} q_{E} / \operatorname{ord}_{p}\left(q_{E}\right)$. As the $j$-invariant $j\left(q_{E}\right)=j(E) \in \mathbb{Q}$ of $E$ is algebraic, $q_{E}$ is transcendental by a theorem of Barré-Sirieix, Diaz, Gramain, and Philibert [1996], and so $\log _{p} q_{E} \neq 0$. Therefore, $\mathfrak{L}\left(V_{f}\right) \neq 0$.

2.3. Iwasawa-Greenberg Selmer groups. Let $f \in S_{k}\left(\Gamma_{0}(N)\right)$ be a newform that is ordinary with respect to an embedding $\mathbb{Q}(f) \hookrightarrow \overline{\mathbb{Q}}_{p}$. Let $L \subset \overline{\mathbb{Q}}_{p}$ be any finite extension of $\mathbb{Q}_{p}$ containing the image of $\mathbb{Q}(f)$ and let $\mathcal{O}$ be the ring of integers of $L$. Let $T_{f} \subset V_{f}$ be a fixed $G_{\mathbb{Q}}$-stable $\mathcal{O}$-lattice.

Let $\Lambda_{\mathcal{O}}=\mathcal{O} \llbracket \Gamma \rrbracket$. Let $\Psi: G_{\mathbb{Q}} \rightarrow \Gamma \subset \Lambda_{\mathcal{O}}^{\times}$be the natural projection. This is a continuous $\Lambda_{\mathcal{O}}$-valued character that is unramified away from $p$ and totally ramified at $p$. Let $\Lambda_{\mathcal{O}}^{*}=\operatorname{Hom}_{\mathrm{cts}}\left(\Lambda_{\mathcal{O}}, \mathbb{Q}_{p} / \mathbb{Z}_{p}\right)$ be the Pontryagin dual of $\Lambda_{\mathcal{O}}$. This 
is a discrete $\Lambda_{\mathcal{O}}$-module via $r \cdot \varphi(x)=\varphi(r x)$, for $r, x \in \Lambda_{\mathcal{O}}$ and $\varphi \in \Lambda_{\mathcal{O}}^{*}$. We similarly define a $\Lambda_{\mathcal{O}}$-module structure on the Pontryagin dual of any $\Lambda_{\mathcal{O}}$-module.

Put $\mathcal{M}=T_{f} \otimes_{\mathcal{O}} \Lambda_{\mathcal{O}}^{*}$, with $G_{\mathbb{Q}}$-action given by $\rho_{f} \otimes \Psi^{-1}$. Let $\mathcal{M}^{+}=T_{f}^{+} \otimes_{\mathcal{O}} \Lambda_{\mathcal{O}}^{*}$ and $\mathcal{M}^{-}=\mathcal{M} / \mathcal{M}^{+}$. Let $\Sigma$ be any finite set of primes containing $p$, and let $S=\Sigma \cup\{\ell \mid N\}$. Let $\mathbb{Q}_{S}$ be the maximal extension of $\mathbb{Q}$ unramified outside $S$ and $\infty$, and let $G_{S}=\operatorname{Gal}\left(\mathbb{Q}_{S} / \mathbb{Q}\right)$. Following Greenberg, we define a Selmer group $\operatorname{Sel}_{\mathbb{Q}_{\infty}, L}^{\Sigma}(f)$ by

$$
\operatorname{Sel}_{\mathbb{Q}_{\infty}, L}^{\Sigma}(f)=\operatorname{ker}\left\{H^{1}\left(G_{S}, \mathcal{M}\right) \rightarrow H^{1}\left(I_{p}, \mathcal{M}^{-}\right)^{G_{\mathbb{Q}_{p}}} \times \prod_{\ell \in S \backslash \Sigma} H^{1}\left(I_{\ell}, \mathcal{M}\right)^{G_{\mathbb{Q}_{\ell}}}\right\} .
$$

This is a discrete, cofinite $\Lambda_{\mathcal{O}}$-module (see [Greenberg 2006, Proposition 3.2]). Its Pontryagin dual $X_{\mathbb{Q}_{\infty}, L}^{\Sigma}(f)$ is a finite $\Lambda_{\mathcal{O}}$-module. We denote by $\mathrm{Ch}_{L}^{\Sigma}(f)$ the $\Lambda_{\mathcal{O}}$-characteristic ideal of $X_{\mathbb{Q}_{\infty}, L}^{\Sigma}(f)$; this is a principal ideal. In general, these all depend on the choice of $T_{f}$, but if $\bar{\rho}_{f}$ is irreducible, then Lemma 2.1.1 shows that $\operatorname{Sel}_{\mathbb{Q}_{\infty}, L}^{\Sigma}(f)$ is independent of $T_{f}$ up to isomorphism, and hence so is $X_{\mathbb{Q}_{\infty}, L}^{\Sigma}(f)$. In particular, if $\bar{\rho}_{f}$ is irreducible, then the ideal $\mathrm{Ch}_{L}^{\Sigma}(f)$ does not depend on the choice of $T_{f}$.

Furthermore, if $L_{1} \supset L$ is a finite extension with ring of integers $\mathcal{O}_{1} \supset \mathcal{O}$, then $T_{f, 1}=T_{f} \otimes_{\mathcal{O}} \mathcal{O}_{1}$ is a $G_{\mathbb{Q}}$-stable $\mathcal{O}_{1}$-lattice in $V_{1}=V_{f} \otimes_{L} L_{1}$ and $T_{f, 1}^{+}=T_{f}^{+} \otimes_{\mathcal{O}} \mathcal{O}_{1}$. Hence $\operatorname{Sel}_{\mathbb{Q}_{\infty}, L_{1}}^{\Sigma}(f)$, the Selmer group defined with respect to the lattice $T_{f, 1}$, is canonically isomorphic to $\operatorname{Sel}_{\mathbb{Q}_{\infty}, L}^{\Sigma}(f) \otimes_{\mathcal{O}} \mathcal{O}_{1}$ as a $\Lambda_{\mathcal{O}_{1}}=\Lambda_{\mathcal{O}} \otimes_{\mathcal{O}} \mathcal{O}_{1}$-module, from which it follows that its Pontryagin dual $X_{\mathbb{Q}_{\infty}, L_{1}}^{\Sigma}(f)$ is isomorphic to $X_{\mathbb{Q}_{\infty}, L}^{\Sigma} \otimes_{\mathcal{O}} \mathcal{O}_{1}$ as a $\Lambda_{\mathcal{O}_{1}}$-module and therefore

$$
\mathrm{Ch}_{L_{1}}^{\Sigma}(f)=\mathrm{Ch}_{L}^{\Sigma}(f) \cdot \Lambda_{\mathcal{O}_{1}} .
$$

The relation between the Selmer groups $\operatorname{Sel}_{\mathbb{Q}_{\infty}, L}^{\Sigma_{1}}(f)$ and $\operatorname{Sel}_{\mathbb{Q}_{\infty}, L}^{\Sigma_{2}}(f)$ with $\Sigma_{1} \subset$ $\Sigma_{2}$ is clear:

$$
\operatorname{Sel}_{\mathbb{Q}_{\infty}, L}^{\Sigma_{1}}(f)=\operatorname{ker}\left\{\operatorname{Sel}_{\mathbb{Q}_{\infty}, L}^{\Sigma_{2}}(f) \stackrel{\text { res }}{\longrightarrow} \prod_{\ell \in S_{2} \backslash S_{1}} H^{1}\left(I_{\ell}, \mathcal{M}\right)^{G_{\mathbb{Q}_{\ell}}}\right\} .
$$

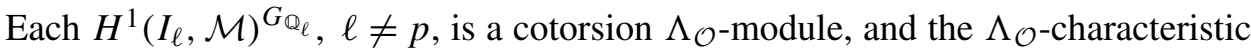
ideal of its Pontryagin dual is generated by $P_{\ell}\left(\Psi^{-1} \epsilon^{-1}\left(\right.\right.$ frob $\left.\left._{\ell}\right)\right)$, where

$$
P_{\ell}(X)=\operatorname{det}\left(1-X \cdot \rho_{f}\left(\operatorname{frob}_{\ell}\right) \mid V_{f, I_{\ell}}\right)
$$

with $V_{f, I_{\ell}}$ being the space of $I_{\ell}$-coinvariants of the representation $V_{f}$. In particular, $X_{\mathbb{Q}_{\infty}, L}^{\Sigma_{2}}(f)$ is a torsion $\Lambda_{\mathcal{O}}$-module if and only if $X_{\mathbb{Q}_{\infty}, L}^{\Sigma_{1}}(f)$ is, and

$$
\mathrm{Ch}_{L}^{\Sigma_{2}}(f) \supseteq \mathrm{Ch}_{L}^{\Sigma_{1}}(f) \cdot \prod_{\ell \in \Sigma_{2} \backslash \Sigma_{1}}\left(P_{\ell}\left(\Psi^{-1} \epsilon^{-1}\left(\text { frob }_{\ell}\right)\right) .\right.
$$

Later, we shall see that this last inclusion is often an equality. 
If $\Sigma=\{p\}$ then we will omit it from our notation, writing $\operatorname{Sel}_{\mathbb{Q}_{\infty}, L}(f), X_{\mathbb{Q}_{\infty}, L}(f)$, and $\mathrm{Ch}_{L}(f)$ instead.

The following lemma shows that if $\Sigma$ is large enough and that if $\bar{\rho}_{f}$ is irreducible, then $\operatorname{Sel}_{\mathbb{Q}_{\infty}, L}^{\Sigma}\left[p^{m}\right]$ and $X_{\mathbb{Q}_{\infty}, L}^{\Sigma}(f) / p^{m} X_{\mathbb{Q}_{\infty}, L}^{\Sigma}(f)$ depend only on the pair ( $T_{f} / p^{m} T_{f}, T_{f}^{+} / p^{m} T_{f}^{+}$) (up to isomorphism).

Lemma 2.3.1. Suppose $\Sigma \supset\{\ell \mid N\}$ and that $\bar{\rho}_{f}$ is irreducible. Then the inclusion $\mathcal{M}\left[p^{m}\right] \subset \mathcal{M}$ induces an identification

$$
\operatorname{Sel}_{\mathbb{Q}_{\infty}, L}^{\Sigma}(f)\left[p^{m}\right]=\operatorname{ker}\left\{H^{1}\left(G_{S}, \mathcal{M}\left[p^{m}\right]\right) \stackrel{\text { res }}{\rightarrow} H^{1}\left(I_{p}, \mathcal{M}^{-}\left[p^{m}\right]\right)^{G_{\mathbb{Q}_{p}}}\right\} .
$$

Since $\mathcal{M}\left[p^{m}\right] \cong T_{f} / p^{m} T_{f} \otimes_{\mathcal{O}} \Lambda_{\mathcal{O}}^{*}\left[p^{m}\right], \mathcal{M}^{+}\left[p^{m}\right] \cong T_{f}^{+} / p^{m} T_{f}^{+} \otimes_{\mathcal{O}} \Lambda_{\mathcal{O}}^{*}\left[p^{m}\right]$, and $\mathcal{M}^{-}\left[p^{m}\right]=\mathcal{M}\left[p^{m}\right] / \mathcal{M}^{+}\left[p^{m}\right]$, it follows that the dependence is only on the pair $\left(T_{f} / p^{m} T_{f}, T_{f}^{+} / p^{m} T_{f}^{+}\right)$.

Proof. Since $\bar{\rho}_{f}$ is irreducible, the inclusion $\mathcal{M}\left[p^{m}\right] \hookrightarrow \mathcal{M}$ induces an identification $H^{1}\left(G_{\Sigma}, \mathcal{M}\left[p^{m}\right]\right)=H^{1}\left(G_{\Sigma}, \mathcal{M}\right)\left[p^{m}\right]$. So $\operatorname{Sel}_{\mathbb{Q}_{\infty}, L}^{\Sigma}(f)\left[p^{m}\right]$ is the kernel of the restriction map $H^{1}\left(G_{\Sigma}, \mathcal{M}\left[p^{m}\right]\right) \rightarrow H^{1}\left(I_{p}, \mathcal{M}^{-}\right)$, which factors through the restriction map $H^{1}\left(G_{\Sigma}, \mathcal{M}\left[p^{m}\right]\right) \rightarrow H^{1}\left(I_{p}, \mathcal{M}^{-}\left[p^{m}\right]\right)$. The kernel of the natural map $H^{1}\left(I_{p}, \mathcal{M}^{-}\left[p^{m}\right]\right) \rightarrow H^{1}\left(I_{p}, \mathcal{M}^{-}\right)$is the image of $\left(\mathcal{M}^{-}\right)^{I_{p}} / p^{m}\left(\mathcal{M}^{-}\right)^{I_{p}}$ via the boundary map. But $\left(\mathcal{M}^{-}\right)^{I_{p}} \cong \operatorname{Hom}_{\mathrm{cts}}\left(\mathcal{O}, \mathbb{Q}_{p} / \mathbb{Z}_{p}\right)$ since $I_{p}$ acts via $\Psi^{-1}$ on $\mathcal{M}^{-} \cong \Lambda_{\mathcal{O}}^{*}$, and so $\left(\mathcal{M}^{-}\right)^{I_{p}} / p^{m}\left(\mathcal{M}^{-}\right)^{I_{p}}=0$ as $\operatorname{Hom}_{\mathrm{cts}}\left(\mathcal{O}, \mathbb{Q}_{p} / \mathbb{Z}_{p}\right)$ is $p$-divisible.

The key to our proofs of both Theorems A and B is an understanding of the images of the restriction maps

$$
H^{1}\left(G_{S}, \mathcal{M}\right) \stackrel{\text { res }}{\rightarrow} H^{1}\left(\mathbb{Q}_{p}, \mathcal{M}^{-}\right) \times \prod_{\ell \in S, \ell \neq p} H^{1}\left(I_{\ell}, \mathcal{M}\right)^{G_{\mathbb{Q}_{\ell}}}
$$

and

$$
H^{1}\left(G_{S}, \mathcal{M}\right) \stackrel{\text { res }}{\longrightarrow} H^{1}\left(I_{p}, \mathcal{M}^{-}\right)^{G_{\mathbb{Q}_{p}}} \times \prod_{\ell \in S, \ell \neq p} H^{1}\left(I_{\ell}, \mathcal{M}\right)^{G_{\mathbb{Q}_{\ell}}},
$$

where $S \supset\{\ell \mid N p\}$ is any finite set of primes. The kernel of (2-3-3) is, of course, just $\operatorname{Sel}_{\mathbb{Q}_{\infty}, L}(f)$. We denote the kernel of (2-3-2) by $\mathcal{S}$ (it is independent of $S$ as $H^{1}\left(G_{S}, \mathcal{M}\right)=\operatorname{ker}\left\{H^{1}\left(G_{S \sqcup\{\ell\}}, \mathcal{M}\right) \stackrel{\text { res }}{\rightarrow} H^{1}\left(I_{\ell}, \mathcal{M}\right)^{\left.\left.G_{Q_{\ell}}\right)\right\}}\right.$ if $\mathcal{M}$ is unramified at $\left.\ell\right)$ and let $\mathcal{X}$ be its Pontryagin dual. As $\mathcal{S}$ is a submodule of each $\operatorname{Sel}_{\mathbb{Q}_{\infty}, L}^{\Sigma}(f), \mathcal{X}$ is a quotient of each $X_{\mathbb{Q}_{\infty}, L}^{\Sigma}(f)$.

The next two propositions record some properties of the above restriction maps. The ideas behind the proofs of these propositions are due to Greenberg (see especially $[1999, \S \S 3,4 ; 2010 b ; 2010 a])$. As there is not a convenient reference for the exact case considered here, we have included the details of the arguments. 
Proposition 2.3.2. Suppose $k \equiv 2(\bmod p-1), \bar{\rho}_{f}$ is irreducible, and $X_{\mathbb{Q}_{\infty}, L}(f)$ is a torsion $\Lambda_{\mathcal{O}}$-module. The restriction maps (2-3-2) and (2-3-3) are surjective.

Proof. As $H^{1}\left(\mathbb{Q}_{p}, \mathcal{M}^{-}\right) \rightarrow H^{1}\left(I_{p}, \mathcal{M}^{-}\right)^{G_{\mathbb{Q}_{p}}},(2-3-3)$ is surjective if (2-3-2) is. That is, to prove the proposition it suffices to prove surjectivity of (2-3-2). To establish this surjectivity we introduce some auxiliary Selmer groups.

Let $\mathcal{N}=\operatorname{Hom}_{\mathcal{O}}\left(T_{f}, \mathcal{O}(1)\right) \otimes_{\mathcal{O}} \Lambda_{\mathcal{O}}$, with $G_{\mathbb{Q}^{-}}$-action given by $\epsilon \rho_{f}^{\vee} \otimes \Psi$, and let $\mathcal{N}^{+}=\operatorname{Hom}_{\mathcal{O}}\left(T_{f} / T_{f}^{+}, \mathcal{O}(1)\right) \otimes_{\mathcal{O}} \Lambda_{\mathcal{O}}$, which is $G_{\mathbb{Q}_{p}}$-stable with $G_{\mathbb{Q}_{p}}$ acting via $\alpha_{f}^{-1} \epsilon \otimes \Psi$. These are free $\Lambda_{\mathcal{O}}$-modules, and $\mathcal{N}^{+}$is a $\Lambda_{\mathcal{O}}$-direct summand of $\mathcal{N}$. Let $\mathcal{N}^{-}=\mathcal{N} / \mathcal{N}^{+}$. The pairing

$$
(\cdot, \cdot): \mathcal{M} \times \mathcal{N} \rightarrow \mathbb{Q}_{p} / \mathbb{Z}_{p}, \quad(t \otimes \varphi, \phi \otimes r)=\varphi(\phi(t) \cdot r),
$$

is a $G_{\mathbb{Q}}$-equivariant perfect pairing under which $\mathcal{M}^{+}$and $\mathcal{N}^{+}$are mutual annihilators. Under the induced (perfect) local Tate pairing

$$
H^{i}\left(\mathbb{Q}_{p}, \mathcal{M}\right) \otimes H^{2-i}\left(\mathbb{Q}_{p}, \mathcal{N}\right) \rightarrow \mathbb{Q}_{p} / \mathbb{Z}_{p},
$$

the images $L_{p}(\mathcal{Y})=\operatorname{im}\left\{H^{1}\left(\mathbb{Q}_{p}, \mathcal{Y}^{+}\right) \rightarrow H^{1}\left(\mathbb{Q}_{p}, \mathcal{Y}\right)\right\}$ for $\mathcal{Y}=\mathcal{M}, \mathcal{N}$ are also mutual annihilators. Let

$$
\operatorname{Sel}^{S}(\mathcal{N})=\operatorname{ker}\left\{H^{1}\left(G_{S}, \mathcal{N}\right) \stackrel{\text { res }}{\longrightarrow} H^{1}\left(\mathbb{Q}_{p}, \mathcal{N}\right) / L_{p}(\mathcal{N}) \hookrightarrow H^{1}\left(\mathbb{Q}_{p}, \mathcal{N}^{-}\right)\right\} .
$$

Let $\amalg^{1}(\mathbb{Q}, S, \mathcal{N}) \subseteq \operatorname{Sel}^{\Sigma}(\mathcal{N})$ consist of those classes that are trivial at all places in $S$.

For $\ell \neq p, H^{1}\left(\mathbb{F}_{\ell}, \mathcal{M}^{I_{\ell}}\right)=0$ and so $H^{1}\left(\mathbb{Q}_{\ell}, \mathcal{M}\right) \stackrel{\sim}{\rightarrow} H^{1}\left(I_{\ell}, \mathcal{M}\right)^{G_{Q_{\ell}}}$. Also, $H^{2}\left(\mathbb{Q}_{p}, \mathcal{M}^{+}\right)=0$ as its dual is $H^{0}\left(\mathbb{Q}_{p}, \mathcal{N}^{-}\right)=0$, so $H^{1}\left(\mathbb{Q}_{p}, \mathcal{M}\right) / L_{p}(\mathcal{M}) \stackrel{\sim}{\longrightarrow}$ $H^{1}\left(\mathbb{Q}_{p}, \mathcal{M}^{-}\right)$. Global Tate duality then identifies the dual of the cokernel of (2-3-2) with $\operatorname{Sel}^{S}(\mathcal{N}) / \amalg^{1}(\mathbb{Q}, S, \mathcal{N})$ (see [Greenberg 2010b, Proposition 3.1]). To show that this last group is trivial, we will prove that $\operatorname{Sel}^{S}(\mathcal{N})$ is $\Lambda_{\mathcal{O}}$-torsion-free if nonzero and also prove that $\operatorname{Sel}^{S}(\mathcal{N})$ is a torsion $\Lambda_{\mathcal{O}}$-module.

Suppose $H^{1}\left(G_{S}, \mathcal{N}\right)$ has nontrivial $\Lambda_{\mathcal{O}}$-torsion: $H^{1}\left(G_{S}, \mathcal{N}\right)[x] \neq 0$ for some $0 \neq y \in \Lambda_{\mathcal{O}}$. Let $\Lambda_{x}=\Lambda_{\mathcal{O}} / x \Lambda_{\mathcal{O}}$ and $N_{x}=\mathcal{N} / x \mathcal{N}$. It follows from the long exact cohomology sequence associated with the short exact sequence

$$
0 \longrightarrow \mathcal{N} \stackrel{n \mapsto x n}{\longrightarrow} \mathcal{N} \longrightarrow N_{x} \longrightarrow 0
$$

that $H^{1}\left(G_{S}, \mathcal{N}\right)[x]$ is the image of $N_{x}^{G_{S}}$ under the boundary map. Let $0 \neq y \in$ $N_{x}^{G_{S}}$. Let $\mathfrak{n} \subset \Lambda_{x}$ be the maximal ideal, and let $r \geq 0$ be the largest integer such that $y \in \mathfrak{n}^{r} N_{x}$. Since $\mathfrak{n}^{r} N_{x} / \mathfrak{n}^{r+1} N_{x} \cong \mathcal{N} \otimes_{\Lambda_{\mathcal{O}}} \mathfrak{n}^{r} / \mathfrak{n}^{r+1}$, the $k\left[G_{S}\right]$-module $N_{x}^{\prime}=\mathfrak{n}^{r} N_{x} / \mathfrak{n}^{r+1} N_{x}$ is just the sum of $\operatorname{dim}_{k}\left(\mathfrak{n}^{r} / \mathfrak{n}^{r+1}\right)$ copies of $\bar{\rho}_{f}$. As $\bar{\rho}_{f}$ is irreducible, it follows that $\left(N_{x}^{\prime}\right)^{G_{S}}=0$. But by the choice of $r, y$ has nontrivial image in $\mathcal{N}_{x}^{\prime}$ and is fixed by $G_{S}$. From this contradiction we conclude ${ }^{5}$ that $H^{1}\left(G_{S}, \mathcal{N}\right)$

${ }^{5}$ See also [Greenberg 2006, Proposition 2.25] for another proof. 
has no nonzero $\Lambda_{\mathcal{O}}$-torsion. The same is then true of the submodules $\operatorname{Sel}^{S}(\mathcal{N})$ and $\amalg^{1}(\mathbb{Q}, S, \mathcal{N})$.

We prove that $\operatorname{Sel}^{S}(\mathcal{N})$ is torsion by exhibiting elements $x$ in the maximal ideal of $\Lambda_{\mathcal{O}}$ such that $\operatorname{Sel}^{S}(\mathcal{N}) / x \operatorname{Sel}^{S}(\mathcal{N})$ has finite order. Let $x=\gamma-u^{m} \in \Lambda_{\mathcal{O}}$ with $m$ an integer. Let $N_{x}=\mathcal{N} / x \mathcal{N}, N_{x}^{+}=\mathcal{N}^{+} / x \mathcal{N}^{+}$, and $N_{x}^{-}=N_{x} / N_{x}^{+}$. These are free $\mathcal{O}$-modules. If $p \nmid N$ or $m \neq 0$, then the natural injection

$$
H^{1}\left(G_{S}, \mathcal{N}\right) / x H^{1}\left(G_{S}, \mathcal{N}\right) \hookrightarrow H^{1}\left(G_{S}, N_{x}\right)
$$

induces an injection

$$
\text { (2-3-4) } \operatorname{Sel}^{S}(\mathcal{N}) / x \operatorname{Sel}^{S}(\mathcal{N}) \hookrightarrow \operatorname{Sel}^{S}\left(N_{x}\right)=\operatorname{ker}\left\{H^{1}\left(G_{S}, N_{x}\right) \rightarrow H^{1}\left(\mathbb{Q}_{p}, N_{x}^{-}\right)\right\} .
$$

For this, we first note that the image of the induced map from $\operatorname{Sel}^{S}(\mathcal{N}) / x \operatorname{Sel}^{S}(\mathcal{N})$ to $H^{1}\left(G_{S}, N_{x}\right)$ lies in $\operatorname{Sel}^{S}\left(N_{x}\right)$. It remains to prove injectivity. Let $c \in \operatorname{Sel}^{S}(\mathcal{N})$ be such that it has trivial image in $\operatorname{Sel}^{S}\left(N_{x}\right)$. Then $c=x d$ for some $d \in H^{1}\left(G_{S}, \mathcal{N}\right)$ such that $x d=0$ in $H^{1}\left(\mathbb{Q}_{p}, \mathcal{N}^{-}\right)$. The kernel of multiplication by $x$ on $H^{1}\left(\mathbb{Q}_{p}, \mathcal{N}^{-}\right)$ is the image of $H^{0}\left(\mathbb{Q}_{p}, N_{x}^{-}\right)$. But $N_{x}^{-}$is a free $\mathcal{O}$-module with $G_{\mathbb{Q}_{p}}$ acting via the character $\alpha_{f} \epsilon^{2-k+m} \omega^{-m}$, and so $H^{0}\left(\mathbb{Q}_{p}, N_{x}^{+}\right)=0$ unless $m=k-2$ and $\alpha_{f}=1$. But $\alpha_{f}=1$ only if $p \| N$ and $k=2$. It follows that if $p \nmid N$ or $m \neq 0$, then multiplication by $x$ is injective on $H^{1}\left(\mathbb{Q}_{p}, \mathcal{N}^{-}\right)$and, therefore, $d \in \operatorname{Sel}^{S}(\mathcal{N})$, proving the injectivity in $(2-3-4)$.

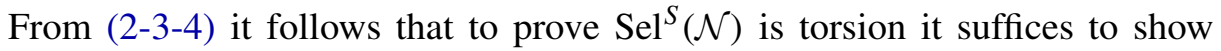
that there is some $m \neq 0$ such that $\operatorname{Sel}^{S}\left(N_{x}\right)$ has finite order. As $\operatorname{Sel}^{S}\left(N_{x}\right)$ has finite order if and only if $\operatorname{Sel}^{S}\left(N_{x}\right) \otimes_{\mathbb{Z}_{p}} \mathbb{Q}_{p} / \mathbb{Z}_{p}$ has finite order - in which case it must be trivial - it suffices to prove the latter. Furthermore, as $\bar{\rho}_{f}$ is irreducible and so $H^{1}\left(G_{S}, N_{x}\right)$ - and hence also $\operatorname{Sel}^{S}\left(N_{x}\right)$ - is a torsion-free $\mathcal{O}$-module and therefore free, it would then follow that $\operatorname{Sel}^{S}\left(N_{x}\right)=0$.

Let $M_{x}=N_{x} \otimes_{\mathbb{Z}_{p}} \mathbb{Q}_{p} / \mathbb{Z}_{p}$ and $M_{x}^{-}=N_{x}^{-} \otimes_{\mathbb{Z}_{p}} \mathbb{Q}_{p} / \mathbb{Z}_{p}$. From the long exact cohomology sequence associated with the short exact sequence

$$
0 \rightarrow N_{x}=N_{x} \otimes_{\mathbb{Z}_{p}} \mathbb{Z}_{p} \rightarrow N_{x} \otimes_{\mathbb{Z}_{p}} \mathbb{Q}_{p} \rightarrow M_{x}=N_{x} \otimes_{\mathbb{Z}_{p}} \mathbb{Q} / \mathbb{Z}_{p} \rightarrow 0
$$

we deduce an injection $H^{1}\left(G_{S}, N_{x}\right) \otimes_{\mathbb{Z}_{p}} \mathbb{Q}_{p} / \mathbb{Z}_{p} \hookrightarrow H^{1}\left(G_{S}, M_{x}\right)$. Under this injection the image of the canonical map

$$
\operatorname{Sel}^{S}\left(N_{x}\right) \otimes_{\mathbb{Z}_{p}} \mathbb{Q}_{p} / \mathbb{Z}_{p} \rightarrow H^{1}\left(G_{S}, N_{x}\right) \otimes_{\mathbb{Z}_{p}} \mathbb{Q}_{p} / \mathbb{Z}_{p}
$$

maps into

$$
\operatorname{Sel}^{S}\left(M_{x}\right)=\operatorname{ker}\left\{H^{1}\left(G_{S}, M_{x}\right) \stackrel{\text { res }}{\rightarrow} H^{1}\left(\mathbb{Q}_{p}, M_{x}^{-}\right)\right\} .
$$

The kernel of the induced map $\operatorname{Sel}^{S}\left(N_{x}\right) \otimes_{\mathbb{Z}_{p}} \mathbb{Q}_{p} / \mathbb{Z}_{p} \rightarrow \operatorname{Sel}^{S}\left(M_{x}\right)$ is then just the kernel of $\operatorname{Sel}^{S}\left(N_{x}\right) \otimes_{\mathbb{Z}_{p}} \mathbb{Q}_{p} / \mathbb{Z}_{p} \rightarrow H^{1}\left(G_{S}, N_{x}\right) \otimes_{\mathbb{Z}_{p}} \mathbb{Q}_{p} / \mathbb{Z}_{p}$, which is finite (having order at most that of the torsion subgroup of the quotient $H^{1}\left(G_{S}, N_{x}\right) / \operatorname{Sel}^{S}\left(N_{x}\right)$ ). 
So to prove that there is an $m \neq 0$ such that $\operatorname{Sel}^{S}\left(N_{x}\right)$ has finite order, it suffices to find such an $m$ for which $\operatorname{Sel}^{S}\left(M_{x}\right)$ has finite order.

Let $m \neq 0$ be an integer such that $m \equiv 0(\bmod p-1)$. Let $y=\gamma-u^{k-2-m}$. Then, as $k \equiv 2(\bmod p-1), M_{x} \cong \mathcal{M}[y]$ as $\mathcal{O}\left[G_{\mathbb{Q}}\right]$-modules, and the isomorphism can be chosen so that $M_{x}^{-}$is identified with $\mathcal{M}^{-}[y]$. It follows that

$$
\operatorname{Sel}^{S}\left(M_{x}\right)=\operatorname{Sel}^{S}(\mathcal{M}[y]) \longleftrightarrow \operatorname{Sel}_{\mathbb{Q}_{\infty}, L}^{S}(f)[y],
$$

where $\operatorname{Sel}^{S}(\mathcal{M}[y])$ is defined just as $\operatorname{Sel}^{S}\left(M_{x}\right)$, and where the injection is induced by the natural identification $H^{1}\left(G_{S}, \mathcal{M}[y]\right) \stackrel{\sim}{\longrightarrow} H^{1}\left(G_{S}, \mathcal{M}\right)[y]$ (which is injective as $\bar{\rho}_{f}$ is irreducible).

As $X_{\mathbb{Q}_{\infty}, L}(f)$ is a torsion $\Lambda_{\mathcal{O}}$-module, so is $X_{\mathbb{Q}_{\infty}, L}^{S}(f)$. Therefore, for all but finitely many integers $m, X_{\mathbb{Q}_{\infty}, L}^{S}(f) / y X_{\mathbb{Q}_{\infty}, L}^{S}(f)$ has finite order. As the latter is dual to $\operatorname{Sel}_{\mathbb{Q}_{\infty}, L}^{S}(f)[y]$, it follows from (2-3-5) that there is an $m \neq 0$ with $m \equiv 0(\bmod p-1)$ such that $\operatorname{Sel}^{S}\left(M_{x}\right)$ has finite order. As explained above, the existence of such an $x$ implies the desired surjectivity of (2-3-2).

Proposition 2.3.3. Suppose $k \equiv 2(\bmod p-1), \bar{\rho}_{f}$ is irreducible, and $X_{\mathbb{Q}_{\infty}, L}(f)$ is a torsion $\Lambda_{\mathcal{O}}$-module.

(i) The $\Lambda_{\mathcal{O}}$-module $\mathcal{X}$ has no nonzero finite-order $\Lambda_{\mathcal{O}}$-submodules.

(ii) Let $\Sigma$ be any finite set of primes containing $p$. The $\Lambda_{\mathcal{O}}$-module $X_{\mathbb{Q}_{\infty}, L}^{\Sigma}(f)$ has no nonzero finite-order $\Lambda_{\mathcal{O}}$-submodules.

Proof. To prove part (i), let $S \supset\{\ell \mid N p\}$ be any finite set of primes and let

$$
\mathcal{P}_{S}=H^{1}\left(\mathbb{Q}_{p}, \mathcal{M}^{-}\right) \times \prod_{\ell \in S, \ell \neq p} H^{1}\left(\mathbb{Q}_{\ell}, \mathcal{M}\right) .
$$

For $x=\gamma-u^{m} \in \Lambda_{\mathcal{O}}, \mathcal{P}_{S}[x]$ is a quotient of

$$
P_{S, x}=H^{1}\left(\mathbb{Q}_{p}, \mathcal{M}[x]\right) / L_{p}(\mathcal{M}[x]) \times \prod_{\ell \in S, \ell \neq p} H^{1}\left(\mathbb{Q}_{\ell}, \mathcal{M}[x]\right),
$$

where $L_{p}(\mathcal{M}[x])=\operatorname{im}\left\{H^{1}\left(\mathbb{Q}_{p}, \mathcal{M}^{+}[x]\right) \rightarrow H^{1}\left(\mathbb{Q}_{p}, \mathcal{M}[x]\right)\right\}$. Therefore the cokernel of the restriction map $H^{1}\left(G_{S}, \mathcal{M}[x]\right)=H^{1}\left(G_{S}, \mathcal{M}\right)[x] \rightarrow \mathcal{P}_{S}[x]$ is a quotient of the cokernel of the restriction map $H^{1}\left(G_{S}, \mathcal{M}[x]\right) \rightarrow P_{S, x}$. By global Tate duality, the Pontryagin dual of the latter is a subquotient of $\operatorname{Sel}^{S}\left(N_{x}\right)$, where $N_{x}$ and $\operatorname{Sel}^{S}\left(N_{x}\right)$ are as in (2-3-4). But, as shown in the proof of Proposition 2.3.2, $m$ can be chosen so that $\operatorname{Sel}^{S}\left(N_{x}\right)=0$ and hence so that $H^{1}\left(G_{S}, \mathcal{M}\right)[x] \rightarrow \mathcal{P}_{S}[x]$. It then follows from an application of the snake lemma to multiplication by $x$ of the short exact sequence

$$
0 \rightarrow \mathcal{S} \rightarrow H^{1}\left(G_{S}, \mathcal{M}\right) \rightarrow \mathcal{P}_{S} \rightarrow 0
$$


that, for such a choice of $m$,

$$
\mathcal{S} / x \mathcal{S} \hookrightarrow H^{1}\left(G_{S}, \mathcal{M}\right) / x H^{1}\left(G_{S}, \mathcal{M}\right) .
$$

However, as shown in both [Skinner and Urban 2014, Lemma 3.3.18] and [Greenberg 2010a, Proposition 2.6.1], the right-hand side of (2-3-6) is trivial for all but finitely many $m$, so the $m$ can also be chosen so that $\mathcal{S} / x \mathcal{S}=0$. Let $X \subseteq \mathcal{X}$ be a sub- $\Lambda_{\mathcal{O}}$-module of finite order, and let $X^{*}$ be its Pontryagin dual. Then $X^{*} / x X^{*}$ is a quotient of $\mathcal{S} / x \mathcal{S}$ and so is 0 . By Nakayama's lemma $X^{*}=0$, hence $X=0$. This proves (i).

To prove part (ii), let $S \supset \Sigma \cup\{\ell \mid N p\}$ and let

$$
\mathcal{P}_{S, \Sigma}=H^{1}\left(I_{p}, \mathcal{M}^{-}\right)^{G_{\mathbb{Q}_{p}}} \times \prod_{\ell \in S \backslash \Sigma} H^{1}\left(\mathbb{Q}_{\ell}, \mathcal{M}\right)
$$

and

$$
\mathcal{P}_{S, \Sigma, x}=H^{1}\left(\mathbb{Q}_{p}, \mathcal{M}[x]\right) / L_{p}(\mathcal{M}[x]) \times \prod_{\ell \in S \backslash \Sigma} H^{1}\left(\mathbb{Q}_{\ell}, \mathcal{M}[x]\right) .
$$

We may then argue as in the proof of part (i) but with $\mathcal{P}_{S}$ replaced by $\mathcal{P}_{S, \Sigma}$. Then $\mathcal{S}$ is replaced by $\operatorname{Sel}_{\mathbb{Q}_{\infty}, L}^{\Sigma}(f)$. Furthermore, as $\mathcal{P}_{S, \Sigma, x}$ is a quotient of $\mathcal{P}_{S, x}$, the surjectivity of the restriction map $H^{1}\left(G_{S}, \mathcal{M}[x]\right) \rightarrow \mathcal{P}_{S, \Sigma, x}$, and hence of the restriction map $H^{1}\left(G_{S}, \mathcal{M}[x]\right) \rightarrow \mathcal{P}_{S, \Sigma}[x]$, follows for a suitable $x=\gamma-u^{m} \in \Lambda_{\mathcal{O}}$ from the surjectivity of the restriction map onto $\mathcal{P}_{S, x}$ established in the proof of part (i).

Let $F_{L}^{\Sigma}(f)$ be the $\Lambda_{\mathcal{O}}$-Fitting ideal of $X_{\mathbb{Q}_{\infty}, L}^{\Sigma}(f)$. The following is a straightforward consequence of the preceding propositions.

Lemma 2.3.4. Suppose $k \equiv 2(\bmod p-1)$ and $\bar{\rho}_{f}$ is irreducible.

(i) $\operatorname{Ch}_{L}^{\Sigma}(f)=\mathrm{Ch}_{L}(f) \cdot \prod_{\ell \in \Sigma, \ell \neq p} P_{\ell}\left(\Psi^{-1} \epsilon^{-1}\left(\right.\right.$ frob $\left.\left._{\ell}\right)\right)$.

(ii) $F_{L}^{\Sigma}(f)=\mathrm{Ch}_{L}^{\Sigma}(f)$.

Proof. If $X_{\mathbb{Q}_{\infty}, L}^{\Sigma}(f)$ is not a torsion $\Lambda_{\mathcal{O}}$-module (equivalently, $X_{\mathbb{Q}_{\infty}, L}(f)$ is not a torsion $\Lambda_{\mathcal{O}}$-module), then $\mathrm{Ch}_{L}(f), \mathrm{Ch}_{L}^{\Sigma}(f)$, and $F_{L}^{\Sigma}(f)$ are all zero, so there is nothing to prove. We suppose then that $X_{\mathbb{Q}_{\infty}, L}^{\Sigma}(f)$ is a torsion $\Lambda_{\mathcal{O}}$-module.

Part (i) is immediate from Proposition 2.3.2 and the definition of characteristic ideals. For part (ii), we first note that $F_{L}^{\Sigma}(f) \subset \mathrm{Ch}_{L}^{\Sigma}(f)$. Let $\mathfrak{a}$ be the kernel of the quotient $\Lambda_{\mathcal{O}} / F_{L}^{\Sigma}(f) \rightarrow \Lambda_{\mathcal{O}} / \mathrm{Ch}_{L}^{\Sigma}(f)$. Since $X_{\mathbb{Q}_{\infty}, L}^{\Sigma}(f)$ is a torsion $\Lambda_{\mathcal{O}}$-module and $\mathrm{Ch}_{L}^{\Sigma}(f)$ is a principal ideal, there exists $\lambda=\gamma-u^{m} \in \Lambda_{\mathcal{O}}$ such that $\lambda$ is not a zero-divisor in $\Lambda_{\mathcal{O}} / \mathrm{Ch}_{L}^{\Sigma}(f)$ and $X_{\mathbb{Q}_{\infty}, L}^{\Sigma}(f) / \lambda X_{\mathbb{Q}_{\infty}, L}^{\Sigma}(f)$ is a torsion $\Lambda_{\mathcal{O}} / \lambda \Lambda_{\mathcal{O}}=\mathcal{O}$ module. The size of this module is then equal to the size of both $\Lambda_{\mathcal{O}} /\left(\lambda, F_{L}^{\Sigma}(f)\right)$ 
and $\Lambda_{\mathcal{O}} /\left(\lambda, \mathrm{Ch}_{L}^{\Sigma}(f)\right)$ (which are necessarily finite), the first by basic properties ${ }^{6}$ of Fitting ideals and the second by Proposition 2.3.3(ii) and a standard argument ${ }^{7}$ from Iwasawa theory. It follows that the natural projection $\Lambda_{\mathcal{O}} /\left(\lambda, F_{L}^{\Sigma}(f)\right) \rightarrow$ $\Lambda_{\mathcal{O}} /\left(\lambda, \mathrm{Ch}_{L}^{\Sigma}(f)\right)$ is an isomorphism. Applying the snake lemma to the diagram obtained by multiplying the short exact sequence

$$
0 \rightarrow \mathfrak{a} \rightarrow \Lambda_{\mathcal{O}} / F_{L}^{\Sigma}(f) \rightarrow \Lambda_{\mathcal{O}} / \mathrm{Ch}_{L}^{\Sigma}(f) \rightarrow 0
$$

by $\lambda$ then yields an exact sequence

$$
0 \rightarrow \mathfrak{a} / \lambda \mathfrak{a} \rightarrow \Lambda_{\mathcal{O}} /\left(\lambda, F_{L}^{\Sigma}(f)\right) \stackrel{\sim}{\longrightarrow} \Lambda_{\mathcal{O}} /\left(\lambda, \mathrm{Ch}_{L}^{\Sigma}(f)\right) \rightarrow 0 .
$$

Therefore $\mathfrak{a} / \lambda \mathfrak{a}$, and hence $\mathfrak{a}$, is 0 .

2.4. p-adic L-functions. Let $f, L, \mathcal{O}$, and $\Lambda_{\mathcal{O}}$ be as in the preceding section, with the assumption that $k \geq 2$ and $f$ is ordinary with respect to $L$. Amice and Vélu [1975] and Vishik [1976] (see also [Mazur et al. 1986]) constructed a $p$ adic $L$-function for $f$. This is a power series $\mathcal{L}_{f} \in \Lambda_{\mathcal{O}}$ with the property that if $\phi: \Lambda_{\mathcal{O}} \rightarrow \overline{\mathbb{Q}}_{p}$ is a continuous $\mathcal{O}$-homomorphism such that $\phi(\gamma)=\zeta u^{m}$ with $\zeta$ a primitive $p^{t_{\phi}-1}$-th root of unity and $0 \leq m \leq k-2$ an integer, then ${ }^{8}$

$$
\begin{aligned}
\mathcal{L}_{f}(\phi) & :=\phi\left(\mathcal{L}_{f}\right)=e(\phi) \frac{p^{t_{\phi}^{\prime}(m+1)} m ! L\left(f, \chi_{\phi}^{-1} \omega^{-m}, m+1\right)}{(-2 \pi i)^{m+1} G\left(\chi_{\phi}^{-1} \omega^{-m}\right) \Omega_{f}^{\operatorname{sgn}\left((-1)^{m}\right)},} \\
e(\phi) & =\alpha_{p}^{-t_{\phi}}\left(1-\frac{\omega^{-m} \chi_{\phi}^{-1} p^{k-2-m}}{\alpha_{p}}\right)\left(1-\frac{\omega^{m} \chi_{\phi}(p) p^{m}}{\alpha_{p}}\right),
\end{aligned}
$$

\footnotetext{
${ }^{6}$ Suppose $R$ is a Noetherian ring and $M$ is a finite $R$-module (hence finitely presented). Let $F_{R}(M)$ be the $R$-Fitting ideal of $M$. These basic properties are: (i) for any ideal $I \subset R, F_{R / I}(M / I M)=$ $F_{R}(M) \bmod I$; (ii) if $M=R / \alpha_{1} \times \cdots \times R / \alpha_{m}$, then $F_{R}(M)=\alpha_{1} \cdots \alpha_{m}$; and (iii) if $R$ is a PID, then length ${ }_{R}(M)=$ length $_{R}\left(R / F_{R}(M)\right)$. For properties (i) and (ii), see [Mazur and Wiles 1984, Appendix A]. Property (iii) follows from (ii).

${ }^{7}$ The argument: A finitely generated torsion $\Lambda_{\mathcal{O}}$-algebra $X$ admits a $\Lambda_{\mathcal{O}}$-homomorphism $X \rightarrow$ $Y=\prod_{i=1}^{r} \Lambda_{\mathcal{O}} /\left(f_{i}\right)$ with finite-order kernel $\mathfrak{a}$ and cokernel $\mathfrak{b}$ and such that the $\Lambda_{\mathcal{O}}$-characteristic ideal of $X$ is $\left(f_{1} \cdots f_{r}\right)$. Let $f=f_{1} \cdots f_{r}$. If $X$ has no finite-order $\Lambda_{\mathcal{O}}$-submodules, then the map to $Y$ is an injection. Multiplying the short exact sequence $0 \rightarrow X \rightarrow Y \rightarrow \mathfrak{b} \rightarrow 0$ by $\lambda=\gamma-u^{m}$ and applying the snake lemma is easily seen to give

$$
\# X / \lambda X=\# Y / \lambda Y=\prod \# \Lambda_{\mathcal{O}} /\left(\lambda, f_{i}\right)=\prod \# \mathcal{O} /\left(f_{i}\left(u^{m}-1\right)\right)=\# \mathcal{O} /\left(f\left(u^{m}-1\right)\right)=\# \Lambda_{\mathcal{O}} /(\lambda, f),
$$

where we have written $f_{i}\left(u^{m}-1\right)$ and $f\left(u^{m}-1\right)$ for the respective images of $f_{i}$ and $f$ under the continuous $\mathcal{O}$-algebra homomorphism $\Lambda_{\mathcal{O}} \rightarrow \mathcal{O}$ sending $\gamma$ to $u^{m}$.

${ }^{8}$ The power of $-2 \pi i$ in the denominator of this formula is incorrectly given as $(-2 \pi i)^{m}$ in some of the formulas in [Skinner and Urban 2014], namely in the introduction, in \$3.4.4, and in Theorem 3.26 of [loc. cit.]. In these cases the correct factor is $(-2 \pi i)^{m+1}$. This error originates in the difference between $\Omega_{f}^{ \pm}$as defined in [loc. cit., §3.3.3] and the $\Omega^{ \pm}$in [Mazur et al. 1986, I.9]: $\Omega^{ \pm}=-2 \pi i \Omega_{f}^{ \pm}$. The exponents of $-2 \pi i$ are correct in the formulas in [Skinner and Urban 2014] for the $L$-function of $f$ twisted by a Hecke character of the imaginary quadratic field $\mathcal{K}$.
} 
where $\alpha_{p}$ is the unique (unit) root in $\mathcal{O}^{\times}$of $x^{2}-a_{p}(f) x+p^{k-1}$ if $p \nmid N$ and $\alpha_{p}=a_{p}(f)$ if $p \mid N, t_{\phi}^{\prime}=0$ if $t_{\phi}=1$ and $p-1 \mid m$ and otherwise $t_{\phi}^{\prime}=t_{\phi}, \chi_{\phi}$ is the primitive Dirichlet character of $p$-power order and conductor (which can be viewed as a finite-order character of $\left.\mathbb{Z}_{p}^{\times}\right)$such that $\chi_{\phi}(u)=\zeta^{-1}, G\left(\chi_{\phi}^{-1} \omega^{-m}\right)$ is the usual Gauss sum (and so equals 1 if $t_{\phi}^{\prime}=0$ ), and $\Omega_{f}^{ \pm}$are the canonical periods of $f$ (these are well defined up to a unit in $\mathcal{O}$; see [Skinner and Urban 2014, §3.3.3]).

Let $\Sigma$ be a finite set of primes. We define an incomplete $p$-adic $L$-function $\mathcal{L}_{f}^{\Sigma} \in \Lambda_{\mathcal{O}}$ by

$$
\mathcal{L}_{f}^{\Sigma}=\mathcal{L}_{f} \cdot \prod_{\ell \in \Sigma, \ell \neq p} P_{\ell}\left(\Psi^{-1} \epsilon^{-1}\left(\operatorname{frob}_{\ell}\right)\right)
$$

Note that

$$
P_{\ell}\left(\Psi^{-1} \epsilon^{-1}\left(\operatorname{frob}_{\ell}\right)\right)= \begin{cases}1-a_{\ell}(f) \ell^{-1} \Psi^{-1}\left(\text { frob }_{\ell}\right)+\ell^{k-3} \Psi^{-2}\left(\text { frob }_{\ell}\right), & \ell \nmid N, \\ 1-a_{\ell}(f) \ell^{-1} \Psi^{-1}\left(\text { frob }_{\ell}\right), & \ell \mid N .\end{cases}
$$

In particular, the value of $\mathcal{L}_{f}^{\Sigma}$ under a continuous $\mathcal{O}$-algebra homomorphism $\phi$ : $\Lambda_{\mathcal{O}} \rightarrow \overline{\mathbb{Q}}_{p}$ such that $\phi(\gamma)=\zeta u^{m}, 0 \leq m \leq k-2$, can be expressed in terms of a special value of an incomplete $L$-function:

$$
\mathcal{L}_{f}^{\Sigma}(\phi)=e(\phi) \frac{p^{t_{\phi}^{\prime}(m+1)} m ! L^{\Sigma \backslash\{p\}}\left(f, \chi_{\phi}^{-1} \omega^{-m}, m+1\right)}{(-2 \pi i)^{m+1} G\left(\chi_{\phi}^{-1} \omega^{-m}\right) \Omega_{f}^{\operatorname{sgn}\left((-1)^{m}\right)}} .
$$

Remark 2.4.1. Let $\mathbb{Z}(f)$ be the ring of integers of $\mathbb{Q}(f)$ and let $\mathfrak{p}$ be the prime of $\mathbb{Z}(f)$ determined by the chosen embedding $\mathbb{Q}(f) \hookrightarrow \overline{\mathbb{Q}}_{p}$. Then $\Omega_{f}^{ \pm}$is well defined up to a unit in the localization $\mathbb{Z}(f)_{(\mathfrak{p})}$ of $\mathbb{Z}(f)$, and the value of the $p$-adic $L$-function under a homomorphism $\phi$ as above lies in a finite extension of $\mathbb{Z}(f)_{(\mathfrak{p})}$. It is in this way that period-normalized values of the $L$-function $L(f, s)$ and its twists, which a priori are complex values, can be viewed as being in $\overline{\mathbb{Q}}_{p}$ without fixing an isomorphism $\overline{\mathbb{Q}}_{p} \cong \mathbb{C}$.

Suppose $f$ has split multiplicative reduction at $p$. Then it follows easily from (2-4-1) that if $\phi_{0}: \Lambda_{\mathcal{O}} \rightarrow \overline{\mathbb{Q}}_{p}$ is the $\mathcal{O}$-algebra homomorphism such that $\phi_{0}(\gamma)=1$, then $\mathcal{L}_{f}\left(\phi_{0}\right)=0$. In particular, $\mathcal{L}_{f}=(\gamma-1) \cdot \mathcal{L}_{f}^{\prime}$ for some $\mathcal{L}_{f}^{\prime} \in \Lambda_{\mathcal{O}}$. Greenberg and Stevens [1993, Theorem 7.1] proved that $\mathcal{L}_{f}^{\prime}\left(\phi_{0}\right)=\phi_{0}\left(\mathcal{L}_{f}^{\prime}\right)$ is related to the $\mathfrak{L}$-invariant of $V_{f}$ by the formula

$$
\mathcal{L}_{f}^{\prime}\left(\phi_{0}\right)=\left(\log _{p} u\right)^{-1} \mathfrak{L}\left(V_{f}\right) \frac{L(f, 1)}{-2 \pi i \Omega_{f}^{+}} .
$$

More precisely, if we identify $\Lambda_{\mathcal{O}}$ with the power-series ring $\mathcal{O} \llbracket T \rrbracket$ by sending $\gamma$ to $1+T$, and if we let $L_{p}(f, s)=\mathcal{L}_{f}\left(u^{s-1}-1\right), s \in \mathbb{Z}_{p}$, then Greenberg and 
Stevens proved that

$$
\left.\frac{d}{d s} L_{p}(f, s)\right|_{s=1}=\mathfrak{L}\left(V_{f}\right) \frac{L(f, 1)}{-2 \pi i \Omega_{f}^{+}} .
$$

This is easily seen to be equivalent to (2-4-3). This formula was conjectured by Mazur, Tate, and Teitelbaum [1986, §13].

2.5. The Iwasawa-Greenberg main conjecture. Let $f, L, \mathcal{O}, \Lambda_{\mathcal{O}}, \mathcal{L}_{f}$, etc., be as in the preceding sections. Along the lines of Iwasawa's original main conjecture for totally real number fields, Mazur and Swinnerton-Dyer (for modular elliptic curves) and Greenberg (more generally) made the following conjecture.

Conjecture 2.5.1. If $\Sigma$ is any finite set of primes containing $p$, then $X_{\mathbb{Q}_{\infty}, L}^{\Sigma}(f)$ is a torsion $\Lambda_{\mathcal{O}}$-module and $\mathrm{Ch}_{L}^{\Sigma}(f)=\left(\mathcal{L}_{f}^{\Sigma}\right)$ in $\Lambda_{\mathcal{O}} \otimes_{\mathbb{Z}_{p}} \mathbb{Q}_{p}$ and even in $\Lambda_{\mathcal{O}}$ if $\bar{\rho}_{f}$ is irreducible.

It follows easily from Lemma 2.3.4(i) and (2-4-2) that if this conjecture holds for one set $\Sigma$ then it holds for all sets $\Sigma$. Also, the conjecture with $L$ replaced by any finite extension implies the conjecture for $L$, as can be seen by the observations in Section 2.3 on the relation (2-3-1) between $\mathrm{Ch}_{L}^{\Sigma}(f)$ and $\mathrm{Ch}_{L_{1}}^{\Sigma}(f)$ for a finite extension $L_{1} \supset L$.

In [Skinner and Urban 2014] the following theorem was proved, in combination with results of Kato [2004], which established this conjecture for a large class of modular forms.

\section{Theorem 2.5.2. Suppose}

(i) $k \equiv 2(\bmod p-1)$;

(ii) $\bar{\rho}_{f}$ is irreducible;

(iii) there exists a prime $q \neq p$ such that $q \| N$ and $\bar{\rho}_{f}$ is ramified at $q$;

(iv) $p \nmid N$ (this is automatic if $k \neq 2$ ).

Then for any finite set of primes $\Sigma, X_{\mathbb{Q}_{\infty}, L}^{\Sigma}(f)$ is a torsion $\Lambda_{\mathcal{O}}$-module and $\mathrm{Ch}_{L}^{\Sigma}(f)=\left(\mathcal{L}_{f}^{\Sigma}\right)$ in $\Lambda_{\mathcal{O}}$.

In [Skinner and Urban 2014] an additional hypothesis is required to conclude equality in $\Lambda_{\mathcal{O}}$ and not just in $\Lambda_{\mathcal{O}} \otimes_{\mathbb{Z}_{p}} \mathbb{Q}_{p}$ :

(*) There exists an $\mathcal{O}$-basis of $T_{f}$ such that the image of $\rho_{f}$ contains $\mathrm{SL}_{2}\left(\mathbb{Z}_{p}\right)$.

This hypothesis was included because it is part of the statement of [Kato 2004, Theorem 17.4]. However, a closer reading of the proof of [loc. cit.] shows that all that is necessary is that (a) $\bar{\rho}_{f}$ be irreducible and (b) there exist an element $g \in \operatorname{Gal}\left(\overline{\mathbb{Q}} / \mathbb{Q}\left[\mu_{p^{\infty}}\right]\right)$ such that $T_{f} /\left(\rho_{f}(g)-1\right) T_{f}$ is a free $\mathcal{O}$-module of rank one, 
as we explain in the following paragraph. All references to theorems or sections in the following paragraph are to [Kato 2004] unless otherwise indicated.

Hypothesis $(*)$ intervenes in the proof of Theorem 17.4 through Theorem 15.5(4), which is proved in $\S 13.14$. Hypothesis (a) together with Lemma 2.1.1 of this paper implies that, in the notation of [Kato 2004], the conclusion in $\S 13.14$ that $T_{f}=a \cdot V_{O_{\lambda}}(f)$ for some $a \in F_{\lambda}^{\times}$holds; Lemma 2.1 .1 of this paper can replace the reference to Lemma 14.7 in $§ 13.14$, which is the only explicit use of a basis with an image containing $\mathrm{SL}_{2}\left(\mathbb{Z}_{p}\right)$ in the proof of Theorem 15.5(4). Hypothesis (a) also, of course, ensures that the hypotheses of Theorem 12.4(3) hold, as needed in $\S 13.14$. Hypothesis (b) ensures that the hypotheses of Theorem 13.4(3) hold. The proof of Theorem $15.5(4)$ in $\$ 13.14$ then holds with $(*)$ replaced by the hypotheses (a) and (b) above.

We now check that (a) and (b) hold under the hypotheses of Theorem 2.5.2. Hypothesis (a) is just hypothesis (ii) of the theorem. Hypothesis (b) is satisfied in light of hypothesis (iii) of the theorem: As $q \| N$, the action of $I_{q}$ on $V_{f}$ is nontrivial and unipotent and in particular factors through the tame quotient (this is a consequence of the "local-global" compatibility of the Galois representation $\rho_{f}$ [Carayol 1986, Theorem A]). It follows that $\rho_{f}(\tau)$ is unipotent for any $\tau \in I_{q}$ projecting to a topological generator of the tame quotient and, since $\bar{\rho}_{f}$ is ramified at $q, \bar{\rho}_{f}(\tau) \neq 1$, hence $T_{f} /\left(\rho_{f}(\tau)-1\right) T_{f}$ is a free $\mathcal{O}$-module of rank one. As $\tau \in \operatorname{Gal}\left(\overline{\mathbb{Q}} / \mathbb{Q}\left[\mu_{p^{\infty}}\right]\right)$, condition (b) holds for $g=\tau$.

We also take this opportunity to note that the reference to [Vatsal 2003] in the proof of [Skinner and Urban 2014, Proposition 12.3.6] is not sufficient. It may be that the weight two specialization of the Hida family in [loc. cit.] that has trivial character also has multiplicative reduction at $p$. This case is excluded in [Vatsal 2003], though the ideas in that paper can be extended to this case, as is explained in [Chida and Hsieh 2016]. The reference to [Vatsal 2003, Theorem 1.1] must be augmented by a reference to [Chida and Hsieh 2016, Theorem C].

The purpose of this paper is, of course, to show that hypothesis (iv) can be removed from Theorem 2.5.2.

The main results of [Skinner and Urban 2014] show that for a suitable imaginary quadratic field $K$ and a large enough set $\Sigma$, the equality $\operatorname{Ch}_{L}^{\Sigma}(f) \operatorname{Ch}_{L}^{\Sigma}\left(f \otimes \chi_{K}\right)=$ $\left(\mathcal{L}_{f}^{\Sigma} \mathcal{L}_{f \otimes \chi_{K}}^{\Sigma}\right)$ holds, where $f \otimes \chi_{K}$ is the newform associated with the twist of $f$ by the primitive quadratic Dirichlet character corresponding to $K$. When $p \nmid N$, this equality can be refined to an equality of the individual factors via the inclusions $\mathcal{L}_{f}^{\Sigma} \in \mathrm{Ch}_{L}^{\Sigma}(f)$ and $\mathcal{L}_{f \otimes \chi_{K}}^{\Sigma} \in \mathrm{Ch}_{L}^{\Sigma}\left(f \otimes \chi_{K}\right)$, which are proved in [Kato 2004]. When $p \mid N$, these inclusions do not follow directly from [Kato 2004]; additional arguments are required.

2.6. Hida families. Let $f \in S_{k}\left(\Gamma_{0}(N)\right)$ be a newform that is ordinary with respect to an embedding $\mathbb{Q}(f) \hookrightarrow \overline{\mathbb{Q}}_{p}$. Write $N=p^{r} M$ with $p \nmid M$ (so $r=0$ or 1 by 
Lemma 2.1.2). Let $L \subset \overline{\mathbb{Q}}_{p}$ be any finite extension of $\mathbb{Q}_{p}$ containing the image of $\mathbb{Q}(f)$ and let $\mathcal{O}$ be the ring of integers of $L$. Let $R_{0}=\mathcal{O} \llbracket X \rrbracket$. Hida (see especially $[1986 ; 1988]$ ) proved that there is a finite, local $R_{0}$-domain $R$ and a formal $q$-expansion

$$
\mathbb{f}=\sum_{n=1}^{\infty} a_{n} q^{n} \in R \llbracket q \rrbracket, \quad a_{1}=1,
$$

satisfying

- $R=R_{0}\left[\left\{a_{\ell}: \ell=\right.\right.$ prime $\left.\}\right]$

- if $\phi: R \rightarrow \overline{\mathbb{Q}}_{p}$ is a continuous $\mathcal{O}$-algebra homomorphism such that $\phi(1+X)=$ $(1+p)^{k^{\prime}}$, with $k^{\prime}>2$ and $k^{\prime} \equiv k(\bmod p-1)$, then $\sum_{n=1}^{\infty} \phi\left(a_{n}\right) q^{n}$ is the $q$ expansion of a $p$-stabilized newform, in the sense that there is a newform $f_{\phi} \in$ $S_{k^{\prime}}\left(\Gamma_{0}(M)\right)$ and an embedding $\mathbb{Q}\left(f_{\phi}\right) \hookrightarrow \overline{\mathbb{Q}}_{p}$ such that $\phi\left(a_{\ell}\right)=a_{\ell}\left(f_{\phi}\right)$ for all primes $\ell \neq p$ and $\phi\left(a_{p}\right)$ is the unit root of the polynomial $x^{2}-a_{p}\left(f_{\phi}\right) x+p^{k^{\prime}-1}$;

- there is a continuous $\mathcal{O}$-algebra homomorphism $\phi_{0}: R \rightarrow \mathcal{O}$ such that $\phi_{0}(1+X)=$ $(1+p)^{k}$ and $\phi_{0}\left(a_{\ell}\right)=a_{\ell}(f), \ell \neq p$, and $\phi_{0}\left(a_{p}\right)$ is the unit root of $x^{2}-a_{p}(f) x+$ $p^{k-1}$ if $r=0$ and $\phi_{0}\left(a_{p}\right)=a_{p}(f)$ if $r=1$.

Furthermore, after possibly replacing $L$ with a finite extension, we may assume

- $\mathcal{O}$ is integrally closed in $R$.

Then, as explained by Greenberg and Stevens [1993] (see also [Nekovár̆ and Plater 2000, (1.4.7)]),

- there is an integer $c$ and an $\mathcal{O}$-algebra embedding

$$
R \hookrightarrow R_{c}=\left\{\sum_{i=0}^{\infty} u_{i}(x-k)^{i}: u_{i} \in L, \lim _{i \rightarrow \infty} \operatorname{ord}_{p}\left(u_{i}\right)+c i=+\infty\right\} \subset L \llbracket x \rrbracket
$$

such that the induced embedding of $R_{0}$ sends $1+X$ to the power series expansion of $(1+p)^{x}$ about $x=k$ and $\phi_{0}$ is the homomorphism induced by evaluating at $x=k$.

Then evaluating at $x=k^{\prime}$ for an integer $k^{\prime}>2$ with $k^{\prime} \equiv k\left(\bmod (p-1) p^{c}\right)$ defines a continuous $\mathcal{O}$-algebra homomorphism $\phi_{k^{\prime}}: R \rightarrow L$ such that $\phi_{k}(1+X)=(1+p)^{k^{\prime}}$ with corresponding newform $f_{\phi_{k^{\prime}}} \in S_{k^{\prime}}\left(\Gamma_{0}(M)\right)$. Furthermore, it is clear that given any integer $m>0$, there is an integer $r_{m}>0$ such that if $k^{\prime} \equiv k\left(\bmod (p-1) p^{r_{m}}\right)$, then $\phi_{k^{\prime}} \equiv \phi_{0}\left(\bmod p^{m} \mathcal{O}\right)$; in particular, for all primes $\ell \neq p$

$$
a_{\ell}\left(f_{\phi_{k^{\prime}}}\right) \equiv a_{\ell}(f)\left(\bmod p^{m} \mathcal{O}\right) .
$$

For each integer $m$ we choose such a $k^{\prime}=k_{m}$ and write $f_{m}$ for the corresponding $f_{\phi_{k_{m}}}$. Note that we have chosen $k_{m}>2$ so that $f_{m}$ is a newform of level not divisible by $p$, though $p$ might divide the level of $f$. 
Suppose that $\bar{\rho}_{f}$ is irreducible. Then there is a free rank two $R$-module $\mathbb{T}$ and a continuous Galois representation

$$
\rho_{R}: G_{\mathbb{Q}} \rightarrow \operatorname{Aut}_{R}(\mathbb{T})
$$

that is unramified at each $\ell \nmid p N$ and such that for any such prime trace $\rho_{R}\left(\operatorname{frob}_{\ell}\right)=$ $a_{\ell} \in R$. In particular for $\phi: R \rightarrow \mathcal{O}$ being $\phi_{0}$ or one of the homomorphisms $\phi_{k_{m}}$, $T_{f_{\phi}}=\mathbb{T} \otimes_{R, \phi} \mathcal{O}$ is a $G_{\mathbb{Q}^{-}}$-stable $\mathcal{O}$-lattice in $\mathbb{T} \otimes_{R, \phi} L \cong V_{f_{\phi}}$. Let $T_{f}=T_{f_{\phi_{0}}}$ and $T_{f_{m}}=T_{f_{\phi_{k_{m}}}}$. Since $\phi_{0}$ and $\phi_{m}$ agree modulo $p^{m}$, reduction modulo $p^{m}$ induces identifications

$$
T_{f} / p^{m} T_{f}=\mathbb{T} \otimes_{R, \phi_{0}} \mathcal{O} / p^{m} \mathcal{O}=\mathbb{T} \otimes_{R, \phi_{m}} \mathcal{O} / p^{m} \mathcal{O}=T_{f_{m}} / p^{m} T_{f_{m}}
$$

as $\mathcal{O}\left[G_{\mathbb{Q}}\right]$-modules.

Suppose also that

$$
\alpha_{f}^{-1} \epsilon^{k-1} \not \equiv \alpha_{f}(\bmod \mathfrak{m}) .
$$

This ensures that there is a free rank-one $G_{\mathbb{Q}_{p}}$-stable $R$-summand $\mathbb{T}^{+} \subset \mathbb{T}$ such that for any of the $\phi$ as before, $\mathbb{T}^{+} \otimes_{R, \phi} \mathcal{O}=T_{f_{\phi}}^{+}$. The identification $T_{f} / p^{m} T_{f}=$ $T_{f_{m}} / p^{m} T_{f_{m}}$ induces an identification

$$
T_{f}^{+} / p^{m} T_{f}^{+}=T_{f_{m}}^{+} / p^{m} T_{f_{m}}^{+} .
$$

Greenberg and Stevens [1993] and Kitagawa [1994] and others have shown that the $p$-adic $L$-functions $\mathcal{L}_{f_{\phi}}$ for the forms $f_{\phi}$ arising from a Hida family fit into a "two-variable" $p$-adic $L$-function. In particular, following Emerton, Pollack, and Weston, we have the following.

Proposition 2.6.1 [Emerton et al. 2006, §3 especially Proposition 3.4.3]. Let $\Sigma$ be a finite set of primes containing $p$. If $\bar{\rho}_{f}$ is irreducible, then there exists $\mathcal{L}_{\mathbb{f}}^{\Sigma} \in R \llbracket \Gamma \rrbracket$ such that for each continuous $\mathcal{O}$-algebra homomorphism $\phi: R \rightarrow \overline{\mathbb{Q}}_{p}$ as above, the image of $\mathcal{L}_{\mathbb{T}}^{\Sigma}$ in $R \llbracket \Gamma \rrbracket \otimes_{R, \phi} \phi(R)^{\prime}=\Lambda_{\phi(R)^{\prime}}$ is a multiple of the p-adic L-function $\mathcal{L}_{f_{\phi}}^{\Sigma}$ by a unit in $\phi(R)^{\prime}$.

Here $\phi(R)^{\prime}$ is the integral closure of $\phi(R)$ in its field of fractions (which is a finite extension of $L$ ). In particular, as $\phi_{k_{m}}(R)=\mathcal{O}$, the image of $\mathcal{L}_{\mathfrak{f}}^{\Sigma}$ in $R \llbracket \Gamma \rrbracket \otimes_{R, \phi_{k_{m}}} \mathcal{O}=$ $\Lambda_{\mathcal{O}}$ is just $u_{m} \mathcal{L}_{f_{m}}^{\Sigma}$ for some $u_{m} \in \mathcal{O}^{\times}$. Assuming that $\bar{\rho}_{f}$ is irreducible, for each $m$ we then have an equality of $\Lambda_{\mathcal{O}}$-ideals

$$
\left(\mathcal{L}_{f}^{\Sigma}, p^{m}\right)=\left(\mathcal{L}_{f_{m}}^{\Sigma}, p^{m}\right) \subseteq \Lambda_{\mathcal{O}}
$$

\section{Assembling the pieces}

We can now put together the various objects and results from Section 2 to prove Theorems A and B as indicated in the introduction. We will freely use the notation introduced in Section 2. 
3.1. Proof of Theorem A. Let $f, L, \mathcal{O}$ be as in the statement of Theorem A. In particular, $f \in S_{k}(N)$ is a newform of some weight $k \geq 2$ that is congruent to 2 modulo $p-1$ and some level $N$. Furthermore, if $f=\sum_{n=1}^{\infty} a_{n}(f) q^{n}$ is the $q$-expansion of $f$, then $a_{p}(f) \in \mathcal{O}^{\times}$. If $p \nmid N$, then by Theorem 2.5.2 the IwasawaGreenberg main conjecture is true: for any finite set of primes $\Sigma$ containing $p$, $\mathrm{Ch}_{L}^{\Sigma}(f)=\left(\mathcal{L}_{f}^{\Sigma}\right)$ in $\Lambda_{\mathcal{O}}$. So we assume that $p \mid N$. By Lemma 2.1.2 we then have $N=p M$ with $p \nmid M$ and $k=2$. Let $T_{f} \subset V_{f}$ be a $G_{\mathbb{Q}^{-}}$-stable $\mathcal{O}$-lattice. By Lemma 2.1.1 this lattice is unique up to $L^{\times}$-multiple since $\bar{\rho}_{f}$ is assumed irreducible.

Let $\Sigma \supset\{\ell \mid N\}$ be a finite set of primes. After possibly replacing $L$ with a finite extension, for each integer $m>0$ there exists

(a) a newform $f_{m} \in S_{k_{m}}\left(\Gamma_{0}(M)\right)$ with $\mathbb{Q}\left(f_{m}\right) \subset L, k_{m}>2$, and $k_{m} \equiv 2(\bmod p-1)$ and such that $a_{p}\left(f_{m}\right) \in \mathcal{O}^{\times}$;

(b) a $G_{\mathbb{Q}}$-stable $\mathcal{O}$-lattice $T_{f_{m}} \subset V_{f_{m}}$ and an isomorphism $T_{f} / p^{m} T_{f} \cong T_{f_{m}} / p^{m} T_{f_{m}}$ as $\mathcal{O}\left[G_{\mathbb{Q}}\right]$-modules that identifies $T_{f}^{+} / p^{m} T_{f}^{+}$with $T_{f_{m}}^{+} / p^{m} T_{f_{m}}^{+}$as $\mathcal{O}\left[G_{\mathbb{Q}_{p}}\right]$ modules;

(c) an equality of ideals $\left(\mathcal{L}_{f}^{\Sigma}, p^{m}\right)=\left(\mathcal{L}_{f_{m}}^{\Sigma}, p^{m}\right) \subseteq \Lambda_{\mathcal{O}}$.

The forms $f_{m}$ in (a) are just those defined in the discussion of Hida families in Section 2.6. Then (b) is just (2-6-1) and (2-6-2), and (c) is (2-6-3). Furthermore, we also have

(d) $\bar{\rho}_{f_{m}} \cong \bar{\rho}_{f}$ is irreducible and ramified at some $q \neq p$ such that $q \| M$;

(e) $X_{\mathbb{Q}_{\infty}, L}^{\Sigma}\left(f_{m}\right)$ is a torsion $\Lambda_{\mathcal{O}}$-module and $\operatorname{Ch}_{L}^{\Sigma}\left(f_{m}\right)=\left(\mathcal{L}_{f_{m}}^{\Sigma}\right) \subseteq \Lambda_{\mathcal{O}}$;

(f) $X_{\mathbb{Q}_{\infty}, L}^{\Sigma}\left(f_{m}\right)$ has no nonzero finite-order $\Lambda_{\mathcal{O}}$-submodules, so $F_{L}^{\Sigma}\left(f_{m}\right)=\mathrm{Ch}^{\Sigma}\left(f_{m}\right)$.

Note that (d) follows from (b) and the hypotheses on $N$ and $\bar{\rho}_{f}$ in Theorem A, while (e) and (f) follow from the Iwasawa-Greenberg main conjecture for $f_{m}$ (which holds by (a), (d), and Theorem 2.5.2 since $f_{m}$ is of level $M$ and $p \nmid M$ ) together with Proposition 2.3.3 and Lemma 2.3.4.

From (b) together with Lemma 2.3.1 we conclude that there is a $\Lambda_{\mathcal{O}}$-isomorphism

$$
\operatorname{Sel}_{\mathbb{Q}_{\infty}, L}^{\Sigma}(f)\left[p^{m}\right] \cong \operatorname{Sel}_{\mathbb{Q}_{\infty}, L}^{\Sigma}\left(f_{m}\right)\left[p^{m}\right]
$$

of $\Lambda_{\mathcal{O}}$-modules, and hence, upon taking Pontryagin duals, also a $\Lambda_{\mathcal{O}}$-isomorphism

$$
X_{\mathbb{Q}_{\infty}, L}^{\Sigma}(f) / p^{m} X_{\mathbb{Q}_{\infty}, L}^{\Sigma}(f) \cong X_{\mathbb{Q}_{\infty}, L}^{\Sigma}\left(f_{m}\right) / p^{m} X_{\mathbb{Q}_{\infty}, L}^{\Sigma}\left(f_{m}\right) .
$$

From basic properties of Fitting ideals we then conclude that there is an equality of $\Lambda_{\mathcal{O}}$-ideals

$$
\left(F_{L}^{\Sigma}(f), p^{m}\right)=\left(F_{L}^{\Sigma}\left(f_{m}\right), p^{m}\right) .
$$

Together with (c), (e), and (f) we then have

$$
\left(F_{L}^{\Sigma}(f), p^{m}\right)=\left(\mathcal{L}_{f}^{\Sigma}, p^{m}\right) \subseteq \Lambda_{\mathcal{O}} .
$$


As $\mathcal{L}_{f}$, and hence $\mathcal{L}_{f}^{\Sigma}$, is nonzero by a well-known theorem of Rohrlich [1988, Theorem 1], if $m$ is large enough then $\left(\mathcal{L}_{f}^{\Sigma}, p^{m}\right) \neq p^{m} \Lambda_{\mathcal{O}}$. From this and (3-1-1) it then follows that if $m$ is large enough, then $\left(F_{L}^{\Sigma}(f), p^{m}\right) \neq p^{m} \Lambda_{\mathcal{O}}$ and hence $F_{L}^{\Sigma}(f) \neq 0$. As $F_{L}^{\Sigma}(f) \neq 0, X_{\mathbb{Q}_{\infty}, L}^{\Sigma}(f)$ must be a torsion $\Lambda_{\mathcal{O}}$-module. It then follows from Proposition 2.3.3(ii) and Lemma 2.3.4(ii) that $\mathrm{Ch}_{L}^{\Sigma}(f)=F_{L}^{\Sigma}(f)$. Combining this with (3-1-1) we then conclude that for all integers $m$

$$
\left(\mathrm{Ch}_{L}^{\Sigma}(f), p^{m}\right)=\left(\mathcal{L}_{f}^{\Sigma}, p^{m}\right) \subseteq \Lambda_{\mathcal{O}}
$$

The characteristic ideal $\operatorname{Ch}_{L}^{\Sigma}(f)$ is a principal ideal. Let $\mathcal{C}_{f}^{\Sigma}$ be a generator. From (3-1-2) it follows that for each integer $m$ there is a $u_{m} \in \Lambda_{\mathcal{O}}$ such that

$$
\mathcal{C}_{f}^{\Sigma}-u_{m} \mathcal{L}_{f}^{\Sigma} \in p^{m} \Lambda_{\mathcal{O}}
$$

Let $\varpi$ be a uniformizer of $\mathcal{O}$ and let $e$ be such that $(p)=\left(\varpi^{e}\right)$. As $\mathcal{L}_{f}^{\Sigma} \neq 0$, there exists an integer $m_{0} \geq 0$ such that $\mathcal{L}_{f}^{\Sigma}(f) \in \varpi^{m_{0}} \Lambda_{\mathcal{O}}$, but $\mathcal{L}_{f}^{\Sigma}(f) \notin \varpi^{m_{0}+1} \Lambda_{\mathcal{O}}$. It then follows from (3-1-3) that

$$
u_{m^{\prime}}-u_{m} \in \varpi^{m e-m_{0}} \Lambda_{\mathcal{O}}, \quad m^{\prime} \geq m .
$$

Therefore the sequence $\left\{u_{m}\right\}$ converges in $\Lambda_{\mathcal{O}}$ to an element $u \in \Lambda_{\mathcal{O}}$ such that for all $m, u-u_{m} \in \varpi^{m e-m_{0}} \Lambda_{\mathcal{O}}$. From this and (3-1-3) it follows that

$$
\mathcal{C}_{f}^{\Sigma}-u \mathcal{L}_{f}^{\Sigma} \in \varpi^{m e-m_{0}} \quad \text { for all } m \geq 0,
$$

whence $\mathcal{C}_{f}^{\Sigma}=u \mathcal{L}_{f}^{\Sigma}$. That is $\mathcal{C}_{f}^{\Sigma} \in\left(\mathcal{L}_{f}^{\Sigma}\right)$.

Since $X_{\mathbb{Q}_{\infty}, L}^{\Sigma}(f)$ is a torsion $\Lambda_{\mathcal{O}}$-module, $\mathrm{Ch}_{L}^{\Sigma}(f)$ is nonzero, and so $\mathcal{C}_{f}^{\Sigma} \neq 0$. We may then reverse the roles of $\mathcal{C}_{f}^{\Sigma}$ and $\mathcal{L}_{f}^{\Sigma}$ in the above argument to show that $\mathcal{L}_{f}^{\Sigma} \in\left(\mathcal{C}_{f}^{\Sigma}\right)$. From the two inclusions we then conclude

$$
\left(\mathcal{L}_{f}^{\Sigma}\right)=\left(\mathcal{C}_{f}^{\Sigma}\right)=\mathrm{Ch}_{L}^{\Sigma} \subseteq \Lambda_{\mathcal{O}}
$$

This proves the desired equality, at least for the chosen $L$ and for $\Sigma$ containing all primes $\ell \mid N$. But, as observed in Section 2.5, this implies the desired equality for all sets $\Sigma$ and all possible $L$. That is, the Iwasawa-Greenberg main conjecture holds for $f$ : Theorem 2.5.2 holds without hypothesis (iv).

3.2. Proof of Theorem B. Let $f, L, \mathcal{O}$ be as in the statement of Theorem B. As these also satisfy the hypotheses of Theorem A, $X_{\mathbb{Q}_{\infty}, L}(f)$ is a torsion $\Lambda_{\mathcal{O}}$-module and its $\Lambda_{\mathcal{O}}$-characteristic ideal $\mathrm{Ch}_{L}(f)$ is generated by the $p$-adic $L$-function $\mathcal{L}_{f}$. Furthermore, by Proposition 2.3.3, neither $X_{\mathbb{Q}_{\infty}, L}(f)$ nor $\mathcal{X}$ have a nonzero finiteorder $\Lambda_{\mathcal{O}}$-submodule. To deduce the conclusions of Theorem B from this, we make a close study of $\operatorname{Sel}_{\mathbb{Q}^{\infty}}(f)[\gamma-1]$ and $\mathcal{S}[\gamma-1]$, following Greenberg [1999].

Since $H^{1}\left(\mathbb{F}_{p},\left(\mathcal{M}^{-}\right)^{I_{p}}\right)=\operatorname{ker}\left\{H^{1}\left(\mathbb{Q}_{p}, \mathcal{M}^{-}\right) \rightarrow H^{1}\left(I_{p}, \mathcal{M}^{-}\right)^{G_{\mathbb{Q}_{p}}}\right\}$, it follows from Proposition 2.3.2 - specifically the surjectivity of (2-3-2) - that there is an 
exact sequence

$$
0 \rightarrow \mathcal{S} \rightarrow \operatorname{Sel}_{\mathbb{Q}_{\infty}, L}(f) \rightarrow H^{1}\left(\mathbb{F}_{p},\left(\mathcal{M}^{-}\right)^{I_{p}}\right) \rightarrow 0 .
$$

As $G_{\mathbb{Q}_{p}}$ acts on $\mathcal{M}^{-} \cong \Lambda^{*}$ through the character $\alpha_{f} \Psi^{-1},\left(\mathcal{M}^{-}\right)^{I_{p}} \cong \Lambda^{*}[\gamma-1]=$ $\operatorname{Hom}_{\mathbb{Z}_{p}}\left(\mathcal{O}, \mathbb{Q}_{p} / \mathbb{Z}_{p}\right) \cong L / \mathcal{O}$, with $G_{\mathbb{Q}_{p}}$ acting through the unramified character $\alpha_{f}$. Let

$$
\alpha_{p}=\alpha_{f}\left(\operatorname{frob}_{p}\right) .
$$

Then $H^{1}\left(\mathbb{F}_{p},\left(\mathcal{M}^{-}\right)^{I_{p}}\right)=0$ unless $\alpha_{p}=1$ (i.e., unless $f$ has split multiplicative reduction at $p$ ), in which case it is isomorphic to $L / \mathcal{O}$. Letting $\operatorname{Ch}_{L}(f)^{\prime}$ be the $\Lambda_{\mathcal{O}}$-characteristic ideal of $\mathcal{X}$, it follows that

$$
\mathrm{Ch}_{L}(f)=\mathrm{Ch}_{L}(f)^{\prime} \cdot \begin{cases}(\gamma-1), & f \text { has split multiplicative reduction at } p, \\ 1, & \text { otherwise. }\end{cases}
$$

This reflects the "extra zero" phenomenon in the split multiplicative case observed at the end of Section 2.4. In fact, we then have

$$
\mathrm{Ch}_{L}(f)^{\prime}= \begin{cases}\left(\mathcal{L}_{f}^{\prime}\right), & f \text { has split multiplicative reduction at } p, \\ \left(\mathcal{L}_{f}\right), & \text { otherwise. }\end{cases}
$$

As $\mathcal{X}$ has no nonzero finite-order $\Lambda_{\mathcal{O}}$-submodules, a standard result ${ }^{9}$ in Iwasawa theory gives $\# \mathcal{X} /(\gamma-1) \mathcal{X}=\# \Lambda_{\mathcal{O}} /\left(\gamma-1, \mathrm{Ch}_{L}(f)^{\prime}\right)$. As $\# \mathcal{S}[\gamma-1]=\# \mathcal{X} /(\gamma-1) \mathcal{X}$, we then find

(3-2-1) $\# \mathcal{S}[\gamma-1]= \begin{cases}\# \mathcal{O} /\left(\mathcal{L}_{f}^{\prime}\left(\phi_{0}\right)\right), & f \text { has split multiplicative reduction at } p, \\ \# \mathcal{O} /\left(\mathcal{L}_{f}\left(\phi_{0}\right)\right), & \text { otherwise, }\end{cases}$ where $\phi_{0}: \Lambda_{\mathcal{O}} \rightarrow \mathcal{O}$ is the continuous $\mathcal{O}$-algebra homomorphism sending $\gamma$ to 1 .

Let $\Sigma=\{\ell \mid N p\}$. Let

$$
W=\mathcal{M}[\gamma-1] \cong T_{f} \otimes_{\mathbb{Z}_{p}} \mathbb{Q}_{p} / \mathbb{Z}_{p} \quad \text { and } \quad W^{ \pm}=\mathcal{M}[\gamma-1]^{ \pm} \cong T_{f}^{ \pm} \otimes_{\mathbb{Z}_{p}} \mathbb{Q}_{p} / \mathbb{Z}_{p} .
$$

Let

$$
\mathcal{P}_{\Sigma}=H^{1}\left(\mathbb{Q}_{p}, \mathcal{M}^{-}\right) \times \prod_{\ell \in \Sigma, \ell \neq p} H^{1}\left(\mathbb{Q}_{\ell}, \mathcal{M}\right)
$$

and

$$
P_{\Sigma}=H^{1}\left(\mathbb{Q}_{p}, W\right) / L_{p}(W) \times \prod_{\ell \in \Sigma, \ell \neq p} H^{1}\left(\mathbb{Q}_{\ell}, W\right),
$$

where $L_{p}(W)=\operatorname{im}\left\{H^{1}\left(\mathbb{Q}_{p}, W^{+}\right) \rightarrow H^{1}\left(\mathbb{Q}_{p}, W\right)\right\}$. Let $P_{\Sigma}^{\text {div }}$ be defined just as $P_{\Sigma}$ but with $L_{p}(W)$ replaced by its maximal divisible subgroup $L_{p}(W)^{\text {div }}$. The usual

${ }^{9}$ See note 5 . 
(torsion) Bloch-Kato Selmer group for $T_{f}$ is just

$$
\operatorname{Sel}_{L}(f)=\operatorname{ker}\left\{H^{1}\left(G_{\Sigma}, W\right) \stackrel{\text { res }}{\rightarrow} P_{\Sigma}^{\text {div }}\right\} \text {. }
$$

As the restriction map $H^{1}\left(G_{\Sigma}, \mathcal{M}\right) \rightarrow \mathcal{P}_{\Sigma}$ is surjective by Proposition 2.3.2, we conclude that there is a short exact sequence

$0 \rightarrow \operatorname{Sel}_{L}(f) \rightarrow \mathcal{S}[\gamma-1] \rightarrow \operatorname{im}\left\{H^{1}\left(G_{\Sigma}, W\right) \stackrel{\text { res }}{\longrightarrow} P_{\Sigma}^{\text {div }}\right\} \cap \operatorname{ker}\left\{P_{\Sigma}^{\text {div }} \rightarrow \mathcal{P}_{\Sigma}[\gamma-1]\right\} \rightarrow 0$.

Let $K=\operatorname{ker}\left\{P_{\Sigma}^{\text {div }} \rightarrow \mathcal{P}_{\Sigma}[\gamma-1]\right\}$. We claim that

$$
\# \mathcal{S}[\gamma-1]=\# \operatorname{Sel}_{L}(f) \cdot \# K \text {. }
$$

If $\operatorname{Sel}_{L}(f)$ is infinite, there is nothing to prove since $\operatorname{Sel}_{L}(f) \subset \mathcal{S}[\gamma-1]$. Suppose then that $\operatorname{Sel}_{L}(f)$ is finite. We will show that the restriction map $H^{1}\left(G_{\Sigma}, W\right) \stackrel{\text { res }}{\longrightarrow}$ $P_{\Sigma}^{\text {div }}$ is surjective, from which the claim follows.

By global duality, the cokernel of the restriction map $H^{1}\left(G_{\Sigma}, W\right) \rightarrow P_{\Sigma}^{\text {div }}$ is dual to a subquotient of

$$
\operatorname{Sel}^{\Sigma}\left(T_{f}\right)^{\mathrm{sat}}=\operatorname{ker}\left\{H^{1}\left(G_{\Sigma}, T_{f}\right) \rightarrow H^{1}\left(\mathbb{Q}_{p}, T_{f}\right) / L_{p}\left(T_{f}\right)^{\mathrm{sat}}\right\},
$$

where $L_{p}\left(T_{f}\right)=\operatorname{im}\left\{H^{1}\left(\mathbb{Q}_{p}, T_{f}^{+}\right) \rightarrow H^{1}\left(\mathbb{Q}_{p}, T_{f}\right)\right\}$ and

$$
L_{p}\left(T_{f}\right)^{\mathrm{sat}}=\left\{x \in H^{1}\left(\mathbb{Q}_{p}, T_{f}\right): p^{n} x \in L_{p}\left(T_{f}\right) \text { for some } n \geq 0\right\} .
$$

Here we have used that $T_{f} \cong \operatorname{Hom}_{\mathbb{Z}_{p}}\left(W, \mathbb{Q}_{p} / \mathbb{Z}_{p}(1)\right)$ as an $\mathcal{O}\left[G_{\mathbb{Q}}\right]$-module and that such an isomorphism identifies $L_{p}\left(T_{f}\right)^{\text {sat }}$ and $L_{p}(W)^{\text {div }}$ as mutual annihilators under local Tate duality. Then $\operatorname{Sel}^{\Sigma}\left(T_{f}\right)^{\text {sat }}$ is a torsion-free $\mathcal{O}$-module (as $\bar{\rho}_{f}$ is irreducible) and its $\mathcal{O}$-rank equals the $\mathcal{O}$-corank of $\operatorname{Sel}_{L}(f)$. In fact, $\operatorname{Sel}^{\Sigma}\left(T_{f}\right)^{\text {sat }}=$ $H^{1}\left(G_{\Sigma}, T_{f}\right) \cap H_{f}^{1}\left(\mathbb{Q}, V_{f}\right)$, where

$$
H_{f}^{1}\left(\mathbb{Q}, V_{f}\right)=\operatorname{ker}\left\{H^{1}\left(G_{\Sigma}, V_{f}\right) \stackrel{\text { res }}{\rightarrow} H^{1}\left(\mathbb{Q}_{p}, V_{f}\right) / L_{p}\left(V_{f}\right) \times \prod_{\ell \in \Sigma, \ell \neq p} H^{1}\left(\mathbb{Q}_{\ell}, V_{f}\right)\right\}
$$

and $L_{p}\left(V_{f}\right)=\operatorname{im}\left\{H^{1}\left(\mathbb{Q}_{p}, V_{f}^{+}\right) \rightarrow H^{1}\left(\mathbb{Q}_{p}, V_{f}\right)\right\}$. (So $H_{f}^{1}\left(\mathbb{Q}, V_{f}\right)$ is just the usual characteristic zero Bloch-Kato Selmer group of $V_{f}$.) In particular, the $\mathcal{O}$-rank of $\operatorname{Sel}^{\Sigma}\left(T_{f}\right)^{\text {sat }}$ is the $L$-dimension of $H_{f}^{1}\left(\mathbb{Q}, V_{f}\right)$. The image of $H_{f}^{1}\left(\mathbb{Q}, V_{f}\right)$ in $H^{1}\left(G_{\Sigma}, T_{f} \otimes_{\mathbb{Z}_{p}} \mathbb{Q}_{p} / \mathbb{Z}_{p}\right) \cong H^{1}\left(G_{\Sigma}, W\right)$ is the maximal divisible submodule of $\operatorname{Sel}_{L}(f)$. However, the latter is assumed to be of finite order, so its maximal divisible subgroup is trivial. This proves that $\operatorname{Sel}^{\Sigma}\left(T_{f}\right)^{\text {sat }}=0$ and hence that the restriction map $H^{1}\left(G_{\Sigma}, W\right) \stackrel{\text { res }}{\longrightarrow} P_{\Sigma, x}^{\text {div }}$ is a surjection. The equality (3-2-2) follows.

Put

$$
L^{\mathrm{alg}}(f, 1)=\frac{L(f, 1)}{-2 \pi i \Omega_{f}^{+}} .
$$


Combining (3-2-1) with (3-2-2), the Greenberg-Stevens formula (2-4-3), and the specialization formula for $\mathcal{L}_{f}$ yields

$$
\# \operatorname{Sel}_{L}(f) \cdot \# K= \begin{cases}\# \mathcal{O} /\left(\frac{1}{\log _{p} u} \cdot \mathfrak{L}\left(V_{f}\right) \cdot L^{\mathrm{alg}}(f, 1)\right), & \alpha_{p}=1, \\ \# \mathcal{O}\left(\left(1-\alpha_{p}\right)^{2} \cdot L^{\operatorname{alg}}(f, 1)\right), & \text { otherwise. }\end{cases}
$$

Therefore, to complete the proof Theorem B it remains to express \# $K$ in terms of Tamagawa factors and the $L$-invariant $\mathfrak{L}\left(V_{f}\right)$.

From the definition of $K$,

$$
K=\prod_{\ell \in \Sigma} K_{\ell},
$$

with

$$
K_{\ell}= \begin{cases}\operatorname{ker}\left\{H^{1}\left(\mathbb{Q}_{\ell}, W\right) \rightarrow H^{1}\left(\mathbb{Q}_{\ell}, \mathcal{M}\right)\right\}, & \ell \neq p \\ \operatorname{ker}\left\{H^{1}\left(\mathbb{Q}_{p}, W\right) / L_{p}(W)^{\operatorname{div}} \rightarrow H^{1}\left(\mathbb{Q}_{p}, \mathcal{M}^{-}\right)\right\}, & \ell=p .\end{cases}
$$

If $\ell \neq p$, then $\mathcal{M}^{I_{\ell}}$ is $(\gamma-1)$-divisible and so $H^{1}\left(I_{\ell}, W\right) \hookrightarrow H^{1}\left(I_{\ell}, \mathcal{M}\right)$ and

$$
K_{\ell}=\operatorname{ker}\left\{H^{1}\left(\mathbb{F}_{\ell}, W^{I_{\ell}}\right) \rightarrow H^{1}\left(\mathbb{F}_{\ell}, \mathcal{M}^{I_{\ell}}\right)=0\right\}=H^{1}\left(\mathbb{F}_{\ell}, W^{I_{\ell}}\right) .
$$

Therefore

$$
\# K_{\ell}=\# H^{1}\left(\mathbb{F}_{\ell}, W^{I_{\ell}}\right)=c_{\ell}\left(T_{f}\right),
$$

where $c_{\ell}\left(T_{f}\right)=\# H^{1}\left(\mathbb{F}_{\ell}, W^{I_{\ell}}\right)$ is just the Tamagawa number at $\ell \neq p$ defined by Bloch and Kato for the $p$-adic representation $T_{f}$. Note that $c_{\ell}\left(T_{f}\right)=1$ if $\ell \nmid N$ (i.e., if $T_{f}$ is unramified at $\ell$ ). Hence to complete the proof of Theorem B it remains to express $\# K_{p}$ in terms of $\alpha_{p}$ if $f$ does not have split multiplicative reduction at $p$ (equivalently $\left.\alpha_{p} \neq 1\right)$ and in terms of $\mathfrak{L}\left(V_{f}\right)$ and the Tamagawa number at $p$ of $T_{f}$ otherwise.

Let

$$
c_{p}^{\prime}=\# \operatorname{ker}\left\{H^{1}\left(\mathbb{Q}_{p}, W\right) / L_{p}(W)^{\operatorname{div}} \rightarrow H^{1}\left(\mathbb{Q}_{p}, W\right) / L_{p}(W)\right\}
$$

and

$$
c_{p}^{\prime \prime}=\# \operatorname{ker}\left\{H^{1}\left(\mathbb{Q}_{p}, W\right) / L_{p}(W) \rightarrow H^{1}\left(\mathbb{Q}_{p}, \mathcal{M}\right) / L_{p}(\mathcal{M})\right\}
$$

Then

$$
\# K_{p}=c_{p}^{\prime} c_{p}^{\prime \prime} .
$$

By Tate local duality, $L_{p}(W)$ is dual to $H^{1}\left(\mathbb{Q}_{p}, T_{f}\right) / L_{p}\left(T_{f}\right)$ and $L_{p}(W)^{\operatorname{div}}$ is dual to $H^{1}\left(\mathbb{Q}_{p}, T_{f}\right) / L_{p}\left(T_{f}\right)^{\text {sat }}$. Therefore

$$
c_{p}^{\prime}=\#\left(L_{p}(W) / L_{p}(W)^{\mathrm{div}}\right)=\#\left(L_{p}\left(T_{f}\right)^{\mathrm{sat}} / L_{p}\left(T_{f}\right)\right) .
$$

Since

$$
H^{1}\left(\mathbb{Q}_{p}, T_{f}\right) / L_{p}\left(T_{f}\right) \hookrightarrow H^{1}\left(\mathbb{Q}_{p}, T_{f}^{-}\right)
$$


and

$$
H^{1}\left(\mathbb{Q}_{p}, T_{f}\right) / L_{p}\left(T_{f}\right)^{\mathrm{sat}} \hookrightarrow H^{1}\left(\mathbb{Q}_{p}, V_{f}^{-}\right),
$$

we find that the (injective) image of $L_{p}\left(T_{f}\right)^{\text {sat }} / L_{p}\left(T_{f}\right)$ in $H^{1}\left(\mathbb{Q}_{p}, T_{f}^{-}\right)$is just $\operatorname{im}\left\{H^{1}\left(\mathbb{Q}_{p}, T_{f}\right) / L_{p}\left(T_{f}\right) \hookrightarrow H^{1}\left(\mathbb{Q}_{p}, T_{f}^{-}\right)\right\} \cap \operatorname{ker}\left\{H^{1}\left(\mathbb{Q}_{p}, T_{f}^{-}\right) \rightarrow H^{1}\left(\mathbb{Q}_{p}, V_{f}^{-}\right)\right\}$.

But $H^{1}\left(I_{p}, T_{f}^{-}\right) \hookrightarrow H^{1}\left(I_{p}, V_{f}^{-}\right)$, so

$$
\operatorname{ker}\left\{H^{1}\left(\mathbb{Q}_{p}, T_{f}^{-}\right) \rightarrow H^{1}\left(\mathbb{Q}_{p}, V_{f}^{-}\right)\right\}=H^{1}\left(\mathbb{F}_{p}, T_{f}^{-}\right) .
$$

On the other hand, the boundary map injects the cokernel of $H^{1}\left(\mathbb{Q}_{p}, T_{f}\right) / L_{p}\left(T_{f}\right) \hookrightarrow$ $H^{1}\left(\mathbb{Q}_{p}, T_{f}^{-}\right)$into $H^{2}\left(\mathbb{Q}_{p}, T_{f}^{+}\right)$but sends the subgroup $H^{1}\left(\mathbb{F}_{p}, T_{f}^{-}\right)$to zero (since $\operatorname{Gal}\left(\overline{\mathbb{F}}_{p} / \mathbb{F}_{p}\right)$ has cohomological dimension one $)$. Hence $H^{1}\left(\mathbb{F}_{p}, T_{f}^{-}\right)$is contained in the image of $H^{1}\left(\mathbb{Q}_{p}, T_{f}\right) / L_{p}\left(T_{f}\right) \hookrightarrow H^{1}\left(\mathbb{Q}_{p}, T_{f}^{-}\right)$. It then follows that

$$
L_{p}\left(T_{f}\right)^{\text {sat }} / L_{p}\left(T_{f}\right) \stackrel{\sim}{\longrightarrow} H^{1}\left(\mathbb{F}_{p}, T_{f}^{-}\right) \cong \begin{cases}0, & \alpha_{p}=1, \\ \mathcal{O} /\left(\alpha_{p}-1\right), & \text { otherwise. }\end{cases}
$$

In particular,

$$
c_{p}^{\prime}= \begin{cases}1, & \alpha_{p}=1, \\ \#\left(\mathcal{O} /\left(\alpha_{p}-1\right)\right), & \text { otherwise. }\end{cases}
$$

It remains to deduce the desired expression for $c_{p}^{\prime \prime}$. By definition $c_{p}^{\prime \prime}$ equals $\#\left(\operatorname{im}\left\{H^{1}\left(\mathbb{Q}_{p}, W\right) / L_{p}(W) \hookrightarrow H^{1}\left(\mathbb{Q}_{p}, W^{-}\right)\right\}\right.$

$$
\left.\cap \operatorname{ker}\left\{H^{1}\left(\mathbb{Q}_{p}, W^{-}\right) \rightarrow H^{1}\left(\mathbb{Q}_{p}, \mathcal{M}^{-}\right)\right\}\right) .
$$

Since $H^{2}\left(\mathbb{Q}_{p}, W^{+}\right)$is dual to $H^{0}\left(\mathbb{Q}_{p}, T_{f}^{-}\right)$and the latter is 0 if $\alpha_{p} \neq 1$, we have $H^{1}\left(\mathbb{Q}_{p}, W\right) / L_{p}(W) \stackrel{\sim}{\rightarrow} H^{1}\left(\mathbb{Q}_{p}, W^{-}\right)$if $\alpha_{p} \neq 1$. It follows that in this case

$$
c_{p}^{\prime \prime}=\# \operatorname{ker}\left\{H^{1}\left(\mathbb{Q}_{p}, W^{-}\right) \rightarrow H^{1}\left(\mathbb{Q}_{p}, \mathcal{M}^{-}\right)\right\}=\#\left(\mathcal{M}^{-}\right)^{G_{\mathbb{Q}_{p}}} /(\gamma-1) \cdot\left(\mathcal{M}^{-}\right)^{G_{\mathbb{Q}_{p}}} .
$$

As $I_{p}$ acts on $\mathcal{M}^{-}$through the character $\Psi^{-1}$ and frob $p$ acts on $\left(\mathcal{M}^{-}\right)^{I_{p}}=$ $\mathcal{M}^{-}[\gamma-1] \cong L / \mathcal{O}$ as multiplication by $\alpha_{p}$, we find

$$
c_{p}^{\prime \prime}=\# L / \mathcal{O}\left[\alpha_{p}-1\right]=\# \mathcal{O} /\left(\alpha_{p}-1\right), \quad \alpha_{p} \neq 1 .
$$

Suppose then that $\alpha_{p}=1$. It follows from local duality that $c_{p}^{\prime \prime}$ equals the index of the $\mathcal{O}$-submodule of $H^{1}\left(\mathbb{Q}_{p}, T_{f}^{+}\right)$generated by $\operatorname{ker}\left\{H^{1}\left(\mathbb{Q}_{p}, T_{f}^{+}\right) \hookrightarrow H^{1}\left(\mathbb{Q}_{p}, T_{f}\right)\right\}$ and the annihilator of $\operatorname{ker}\left\{H^{1}\left(\mathbb{Q}_{p}, W^{-}\right) \rightarrow H^{1}\left(\mathbb{Q}_{p}, \mathcal{M}^{-}\right)\right\}$. The first is just the image of $\mathcal{O} \cong H^{0}\left(\mathbb{Q}_{p}, T_{f}^{-}\right) \rightarrow H^{1}\left(\mathbb{Q}_{p}, T_{f}^{+}\right)$determined by the $G_{\mathbb{Q}_{p}}$-extension $T_{f}$. Let $c_{V_{f}}$ be an $\mathcal{O}$-generator; this is a nonzero element in $\ell_{V_{f}}$ in the notation of Section 2.2. On the other hand, as $H^{1}\left(\mathbb{Q}_{p}, W^{-}\right) \cong \operatorname{Hom}_{\mathrm{cts}}\left(G_{\mathbb{Q}_{p}}^{\mathrm{ab}, p}, L / \mathcal{O}\right)$, the kernel 
$\operatorname{ker}\left\{H^{1}\left(\mathbb{Q}_{p}, W^{-}\right) \rightarrow H^{1}\left(\mathbb{Q}_{p}, \mathcal{M}^{-}\right)\right\}$is readily seen to be $\operatorname{Hom}_{\mathrm{cts}}(\Gamma, L / \mathcal{O})$ - those homomorphisms that factor through $\Gamma$. Then, under the identification

$$
H^{1}\left(\mathbb{Q}_{p}, T_{f}^{+}\right)=H^{1}\left(\mathbb{Q}_{p}, \mathcal{O}(1)\right)=\left({\underset{\lim }{n}}_{\mathbb{Q}_{p}} \times /\left(\mathbb{Q}_{p}{ }^{\times}\right)^{p^{n}}\right) \otimes_{\mathbb{Z}_{p}} \mathcal{O}
$$

the annihilator of $\operatorname{Hom}_{\mathrm{cts}}(\Gamma, L / \mathcal{O})$ is identified with the $\mathcal{O}$-module $p \otimes \mathcal{O}$ generated by the image of $p^{\mathbb{Z}}$. The index of $\mathcal{O} \cdot c_{V_{f}}+p \otimes \mathcal{O}$ is just the index of the projection of $c_{V_{f}}$ to $\left(\lim _{n} \mathbb{Z}_{p}^{\times} /\left(\mathbb{Z}_{p}^{\times}\right)^{p^{n}}\right) \otimes_{\mathbb{Z}_{p}} \mathcal{O}$. From the definition of $\psi_{\text {cyc }}$ in Section 2.2, this index is just $\# \mathcal{O} /\left(\left(1 / \log _{p} u\right) \cdot \psi_{\text {cyc }}\left(c_{V_{f}}\right)\right)$. So by the definition of $\psi_{\text {ur }}$ (which is nonzero on $c_{V_{f}}$ as $\left.0 \neq c_{V_{f}} \in \ell_{V_{f}}\right)$ and the definition of $\mathfrak{L}\left(V_{f}\right)$,

$c_{p}^{\prime \prime}=\# \mathcal{O} /\left(\left(1 / \log _{p} u\right) \cdot \psi_{\mathrm{cyc}}\left(c_{V_{f}}\right)\right)=\# \mathcal{O} /\left(\left(1 / \log _{p} u\right) \cdot \mathfrak{L}\left(V_{f}\right) \cdot \psi_{\mathrm{ur}}\left(c_{V_{f}}\right)\right), \quad \alpha_{p}=1$.

Combining the formulas for $c_{p}^{\prime \prime}$ in the two cases with those for $c_{p}^{\prime}$ we find

$$
\# K_{p}= \begin{cases}\# \mathcal{O} /\left(\left(1 / \log _{p} u\right) \cdot \mathfrak{L}\left(V_{f}\right) \cdot \psi_{\mathrm{ur}}\left(c_{V_{f}}\right)\right), & \alpha_{p}=1, \\ \# \mathcal{O} /\left(\alpha_{p}-1\right)^{2}, & \alpha_{p} \neq 1 .\end{cases}
$$

Suppose $\mathfrak{L}\left(V_{f}\right) \neq 0$ if $\alpha_{p}=1$. Then combining (3-2-3) with (3-2-4), (3-2-5), and (3-2-6) yields

$$
\# \mathcal{O} /\left(L^{\mathrm{alg}}(f, 1)\right)=\# \operatorname{Sel}_{L}(f) \cdot \prod_{\ell \neq p} c_{\ell}\left(T_{f}\right) \cdot \begin{cases}\# \mathcal{O} /\left(\psi_{\mathrm{ur}}\left(c_{V_{f}}\right)\right), & \alpha_{p}=1, \\ 1, & \alpha_{p} \neq 1 .\end{cases}
$$

That the final term is just the Bloch-Kato Tamagawa number at $p$ of the representation $T_{f}$, which we denote $c_{p}\left(T_{f}\right)$, can be shown as in [Dummigan 2005] (in that paper $c_{p}\left(T_{f}\right)$ is denoted $\operatorname{Tam}_{M}^{0}\left(T_{f}\right)$ ). The only significant change is the need to include the $\mathcal{O}$-action, but this is a straightforward modification. In the $p \nmid N$ casethat is, the case where $V_{f}$ is a crystalline representation of $G_{\mathbb{Q}_{p}}$ - the fact that $c_{p}\left(T_{f}\right)=1$ follows by the arguments used to prove [Dummigan 2005, Theorem 5.1]. The $p \| N$ case - in which case $V_{f}$ is a semistable representation of $G_{\mathbb{Q}_{p}}$ - follows as in [Dummigan 2005, §7] from the arguments used to prove [Dummigan 2005, Theorem 6.1]. We therefore have the formula asserted in Theorem B:

$$
\# \mathcal{O} /\left(L^{\operatorname{alg}}(f, 1)\right)=\# \operatorname{Sel}_{L}(f) \cdot \prod_{\ell} c_{\ell}\left(T_{f}\right) .
$$

This completes the proof of Theorem B.

3.3. Proof of Theorem $C$. Theorem $C$ is just a special case of Theorem B. To see this, let $E$ be as in Theorem $\mathrm{C}$ and let $f \in S_{2}\left(\Gamma_{0}(N)\right)$ be the newform associated with $E$, so $N$ is the conductor of $E$ and $L(E, s)=L(f, s)$. For Theorem C to follow from Theorem B, it suffices to have that under the hypotheses of Theorem $\mathrm{C}$, hypotheses (i), (ii), and (iii) of Theorem B hold for $f$ and $\Omega_{E}$ is a $\mathbb{Z}_{(p)}^{\times}$-multiple of $-2 \pi i \Omega_{f}^{+}$. 
That hypotheses (i) and (ii) of Theorem C imply hypotheses (i) and (ii) of Theorem B is immediate. Furthermore, as noted in the example at the end of Section 2.2, if $E$ has split multiplicative reduction at $p$ then the $\mathfrak{L}$-invariant $\mathfrak{L}\left(V_{f}\right)$ of $f$ is nonzero, hence hypothesis (iii) of Theorem $\mathrm{B}$ also holds.

To compare periods, we first recall that if $\omega_{E}$ is a Néron differential of $E$ then

$$
\Omega_{E}=\int_{c^{+}} \omega_{E} \in \mathbb{C}^{\times}
$$

where $c^{+}$is a generator of the submodule $H_{1}(E(\mathbb{C}), \mathbb{Z})^{+} \subset H_{1}(E(\mathbb{C}), \mathbb{Z})$ that is fixed by the action of $\operatorname{Gal}(\mathbb{C} / \mathbb{R})$; this is well defined up to multiplication by \pm 1 . Now let

$$
\phi: X_{1}(N) \rightarrow E^{\mathrm{opt}}
$$

be an optimal parametrization for the $\mathbb{Q}$-isogeny class of $E$ as in [Stevens 1989, Proposition (1.4)]. Then, as demonstrated in the proof of [Greenberg and Vatsal 2000, Proposition (3.1)], $\Omega_{E^{\text {opt }}}$ equals $-2 \pi i \Omega_{f}^{+}$up to a $\mathbb{Z}_{(p)}^{\times}$-multiple $^{10}$. Let

$$
\beta: E^{\mathrm{opt}} \rightarrow E
$$

be a $\mathbb{Q}$-isogeny. Since $E[p]$ is an irreducible $G_{\mathbb{Q}}$-representation, $\beta$ can be chosen so that its degree is prime to $p$. Then $\beta^{*} \omega_{E}$ is a $\mathbb{Z}_{(p)}^{\times}$-multiple of $\omega_{E \text { opt }}$, and so $\Omega_{E}$ is a $\mathbb{Z}_{(p)}^{\times}$-multiple of $\Omega_{E^{\text {opt }}}$ and hence also of $-2 \pi i \Omega_{f}^{+}$.

\section{Acknowledgments}

The author thanks Xin Wan for helpful conversations and Manjul Bhargava for helpful comments. The author's work was partially supported by the grants DMS0758379 and DMS-1301842 from the National Science Foundation.

\section{References}

[Amice and Vélu 1975] Y. Amice and J. Vélu, "Distributions $p$-adiques associées aux séries de Hecke”, pp. 119-131 in Journées Arithmétiques de Bordeaux (Univ. Bordeaux, Bordeaux, 1974), Astérisque 24-25, Soc. Math., Paris, 1975. MR 0376534 Zbl 0332.14010

[Barré-Sirieix et al. 1996] K. Barré-Sirieix, G. Diaz, F. Gramain, and G. Philibert, "Une preuve de la conjecture de Mahler-Manin”, Invent. Math. 124:1-3 (1996), 1-9. MR 1369409 Zbl 0853.11059

[Bloch and Kato 1990] S. Bloch and K. Kato, "L-functions and Tamagawa numbers of motives", pp. 333-400 in The Grothendieck Festschrift, vol. I, edited by P. Cartier et al., Progr. Math. 86, Birkhäuser, Boston, 1990. MR 1086888 Zbl 0768.14001

${ }^{10}$ The key points are [Greenberg and Vatsal 2000, Proposition (3.3)], which shows that if $\operatorname{ord}_{p}(N) \leq 1$ then $\phi^{*} \omega_{E^{\text {opt }}}=c \cdot 2 \pi i f(z) d z$ for some integer $c \in \mathbb{Z}$ such that $p \nmid c$, and the factby the definition of an optimal parametrization - that $\phi$ induces a surjection $H_{1}\left(X_{1}(N)(\mathbb{C}), \mathbb{Z}\right) \rightarrow$ $H_{1}\left(E^{\mathrm{opt}}(\mathbb{C}), \mathbb{Z}\right)$. 
[Carayol 1986] H. Carayol, "Sur les représentations $l$-adiques associées aux formes modulaires de Hilbert”, Ann. Sci. École Norm. Sup. (4) 19:3 (1986), 409-468. MR 870690 Zbl 0616.10025

[Chida and Hsieh 2016] M. Chida and M.-L. Hsieh, "Special values of anticyclotomic $L$-functions for modular forms", J. Reine Angew. Math. (online publication January 2016).

[Dummigan 2005] N. Dummigan, "Tamagawa factors for certain semi-stable representations", Bull. London Math. Soc. 37:6 (2005), 835-845. MR 2186716 Zbl 1137.11325

[Emerton et al. 2006] M. Emerton, R. Pollack, and T. Weston, "Variation of Iwasawa invariants in Hida families", Invent. Math. 163:3 (2006), 523-580. MR 2207234 Zbl 1093.11065

[Fouquet 2014] O. Fouquet, "The Equivariant Tamagawa Number Conjecture for modular motives with coefficients in the Hecke algebra", preprint, 2014. arXiv 1401.1715

[Greenberg 1999] R. Greenberg, "Iwasawa theory for elliptic curves", pp. 51-144 in Arithmetic theory of elliptic curves (Cetraro, 1997), edited by C. Viola, Lecture Notes in Math. 1716, Springer, Berlin, 1999. MR 1754686 Zbl 0946.11027

[Greenberg 2006] R. Greenberg, "On the structure of certain Galois cohomology groups", Doc. Math. Extra Vol. (2006), 335-391. MR 2290593

[Greenberg 2010a] R. Greenberg, "On the structure of Selmer groups", preprint, 2010, Available at https://www.math.washington.edu/ greenber/Sel.pdf.

[Greenberg 2010b] R. Greenberg, "Surjectivity of the global-to-local map defining a Selmer group", Kyoto J. Math. 50:4 (2010), 853-888. MR 2740696 Zbl 1230.11133

[Greenberg and Stevens 1993] R. Greenberg and G. Stevens, " $p$-adic $L$-functions and $p$-adic periods of modular forms”, Invent. Math. 111:2 (1993), 407-447. MR 1198816 Zbl 0778.11034

[Greenberg and Vatsal 2000] R. Greenberg and V. Vatsal, "On the Iwasawa invariants of elliptic curves”, Invent. Math. 142:1 (2000), 17-63. MR 1784796 Zbl 1032.11046

[Hida 1986] H. Hida, "Galois representations into $\mathrm{GL}_{2}\left(\mathbf{Z}_{p}[[X]]\right)$ attached to ordinary cusp forms", Invent. Math. 85:3 (1986), 545-613. MR 848685 Zbl 0612.10021

[Hida 1988] H. Hida, "A $p$-adic measure attached to the zeta functions associated with two elliptic modular forms, II”, Ann. Inst. Fourier (Grenoble) 38:3 (1988), 1-83. MR 976685 Zbl 0645.10028

[Kato 2004] K. Kato, " $p$-adic Hodge theory and values of zeta functions of modular forms", pp. ix, 117-290 in Cohomologies p-adiques et applications arithmétiques, III, edited by P. Berthelot et al., Astérisque 295, Société Mathématique de France, Paris, 2004. MR 2104361 Zbl 1142.11336

[Kitagawa 1994] K. Kitagawa, "On standard p-adic $L$-functions of families of elliptic cusp forms", pp. 81-110 in p-adic monodromy and the Birch and Swinnerton-Dyer conjecture (Boston, 1991), edited by B. Mazur and G. Stevens, Contemp. Math. 165, Amer. Math. Soc., Providence, RI, 1994. MR 1279604 Zbl 0841.11028

[Mazur and Wiles 1984] B. Mazur and A. Wiles, "Class fields of abelian extensions of Q", Invent. Math. 76:2 (1984), 179-330. MR 742853 Zbl 0545.12005

[Mazur et al. 1986] B. Mazur, J. Tate, and J. Teitelbaum, "On $p$-adic analogues of the conjectures of Birch and Swinnerton-Dyer”, Invent. Math. 84:1 (1986), 1-48. MR 830037 Zbl 0699.14028

[Miyake 1989] T. Miyake, Modular forms, Springer, Berlin, 1989. Translation of Hokei keishiki to seisūron Kinokuniya Company, Tokyo, 1976. MR 1021004 Zbl 0701.11014

[Nekovář and Plater 2000] J. Nekovář and A. Plater, "On the parity of ranks of Selmer groups", Asian J. Math. 4:2 (2000), 437-497. MR 1797592 Zbl 0973.11066

[Rohrlich 1988] D. E. Rohrlich, "L-functions and division towers", Math. Ann. 281:4 (1988), 611632. MR 958262 Zbl 0656.14013 
[Saito 1997] T. Saito, "Modular forms and p-adic Hodge theory", Invent. Math. 129:3 (1997), 607-620. MR 1465337 Zbl 0877.11034

[Skinner and Urban 2014] C. Skinner and E. Urban, "The Iwasawa main conjectures for GL 2 ", Invent. Math. 195:1 (2014), 1-277. MR 3148103 Zbl 1301.11074

[Stevens 1989] G. Stevens, "Stickelberger elements and modular parametrizations of elliptic curves", Invent. Math. 98:1 (1989), 75-106. MR 1010156 Zbl 0697.14023

[Vatsal 2003] V. Vatsal, "Special values of anticyclotomic L-functions", Duke Math. J. 116:2 (2003), 219-261. MR 1953292 Zbl 1065.11048

[Višik 1976] M. M. Višik, "Nonarchimedean measures associated with Dirichlet series", Mat. Sb. (N.S.) 99(141): 2 (1976), 248-260, 296. In Russian; translated in Mathematics of the USSR-Sbornik 28:2 (1976), 216-228. MR 0412114

[Wiles 1988] A. Wiles, "On ordinary $\lambda$-adic representations associated to modular forms", Invent. Math. 94:3 (1988), 529-573. MR 969243 Zbl 0664.10013

[Zhang 2014] W. Zhang, "Selmer groups and the indivisibility of Heegner points", Camb. J. Math. 2:2 (2014), 191-253. MR 3295917

Received May 18, 2014. Revised December 23, 2014.

CHRISTOPHER SKINNER

Department OF MATHEMATiCS

PRINCETON UNIVERSITY

Fine Hall, WASHINGTON RoAD

PRINCETON, NJ 08544-1000

UNITED STATES

cmcls@princeton.edu 


\title{
PACIFIC JOURNAL OF MATHEMATICS
}

Founded in 1951 by E. F. Beckenbach (1906-1982) and F. Wolf (1904-1989)

$$
\text { msp.org/pjm }
$$

\section{EDITORS}

\author{
Don Blasius (Managing Editor) \\ Department of Mathematics \\ University of California \\ Los Angeles, CA 90095-1555 \\ blasius@math.ucla.edu
}

\author{
Paul Balmer \\ Department of Mathematics \\ University of California \\ Los Angeles, CA 90095-1555 \\ balmer@math.ucla.edu \\ Robert Finn \\ Department of Mathematics \\ Stanford University \\ Stanford, CA 94305-2125 \\ finn@math.stanford.edu \\ Sorin Popa \\ Department of Mathematics \\ University of California \\ Los Angeles, CA 90095-1555 \\ popa@math.ucla.edu
}

\author{
Vyjayanthi Chari \\ Department of Mathematics \\ University of California \\ Riverside, CA 92521-0135 \\ chari@math.ucr.edu \\ Kefeng Liu \\ Department of Mathematics \\ University of California \\ Los Angeles, CA 90095-1555 \\ liu@math.ucla.edu \\ Igor Pak \\ Department of Mathematics \\ University of California \\ Los Angeles, CA 90095-1555 \\ pak.pjm@gmail.com \\ Paul Yang \\ Department of Mathematics \\ Princeton University \\ Princeton NJ 08544-1000 \\ yang@math.princeton.edu
}

\section{PRODUCTION}

Silvio Levy, Scientific Editor, production@msp.org

\section{SUPPORTING INSTITUTIONS}

ACADEMIA SINICA, TAIPEI

CALIFORNIA INST. OF TECHNOLOGY

STANFORD UNIVERSITY

UNIV. OF BRITISH COLUMBIA

UNIV. OF CALIFORNIA, BERKELEY

UNIV. OF CALIFORNIA, DAVIS

UNIV. OF CALIFORNIA, LOS ANGELES

UNIV. OF CALIFORNIA, RIVERSIDE

UNIV. OF CALIFORNIA, SAN DIEGO

UNIV. OF CALIF., SANTA BARBARA
KEIO UNIVERSITY

MATH. SCIENCES RESEARCH INSTITUTE

NEW MEXICO STATE UNIV.

OREGON STATE UNIV.
Daryl Cooper

Department of Mathematics

University of California

Santa Barbara, CA 93106-3080 cooper@math.ucsb.edu

Jiang-Hua Lu

Department of Mathematics

The University of Hong Kong

Pokfulam Rd., Hong Kong

jhlu@maths.hku.hk

$$
\text { Jie Qing }
$$

Department of Mathematics

University of California

Santa Cruz, CA 95064

qing@cats.ucsc.edu

\author{
UNIV. OF CALIF., SANTA CRUZ \\ UNIV. OF MONTANA \\ UNIV. OF OREGON \\ UNIV. OF SOUTHERN CALIFORNIA \\ UNIV. OF UTAH \\ UNIV. OF WASHINGTON \\ WASHINGTON STATE UNIVERSITY
}

These supporting institutions contribute to the cost of publication of this Journal, but they are not owners or publishers and have no responsibility for its contents or policies.

See inside back cover or msp.org/pjm for submission instructions.

The subscription price for 2016 is US \$/year for the electronic version, and \$/year for print and electronic.

Subscriptions, requests for back issues and changes of subscriber address should be sent to Pacific Journal of Mathematics, P.O. Box 4163, Berkeley, CA 94704-0163, U.S.A. The Pacific Journal of Mathematics is indexed by Mathematical Reviews, Zentralblatt MATH, PASCAL CNRS Index, Referativnyi Zhurnal, Current Mathematical Publications and Web of Knowledge (Science Citation Index).

The Pacific Journal of Mathematics (ISSN 0030-8730) at the University of California, c/o Department of Mathematics, 798 Evans Hall \#3840, Berkeley, CA 94720-3840, is published twelve times a year. Periodical rate postage paid at Berkeley, CA 94704, and additional mailing offices. POSTMASTER: send address changes to Pacific Journal of Mathematics, P.O. Box 4163, Berkeley, CA 94704-0163.

PJM peer review and production are managed by EditFLOW ${ }^{\circledR}$ from Mathematical Sciences Publishers.

PUBLISHED BY

\section{I. mathematical sciences publishers}

nonprofit scientific publishing

http://msp.org/

(C) 2016 Mathematical Sciences Publishers 


\section{PACIFIC JOURNAL OF MATHEMATICS}

Volume $283 \quad$ No. $1 \quad$ July 2016

A New family of simple $\mathfrak{g l}_{2 n}(\mathbb{C})$-modules

JONATHAN NILSSON

Derived categories of representations of small categories over commutative noetherian rings

BENJAMIN ANTIEAU and GREg STEVENSON

Vector bundles over a real elliptic curve

INDRANIL BISWAS and FLORENT SCHAFFHAUSER

$\mathrm{Q}(\mathrm{N})$-graded Lie superalgebras arising from fermionic-bosonic representations

JIN CHENG

Conjugacy and element-conjugacy of homomorphisms of compact Lie groups

YiNGJUE FANG, GANG HAN and BINYONG SUN

Entire sign-changing solutions with finite energy to the fractional Yamabe equation

DANILO GARRIDO and MoniCa Musso

Calculation of local formal Mellin transforms

ADAM GRAHAM-SQUIRE

The untwisting number of a knot

KENAN INCE

A Plancherel formula for $L^{2}(G / H)$ for almost symmetric subgroups

Bent Ørsted and Birgit SPEH

Multiplicative reduction and the cyclotomic main conjecture for $\mathrm{GL}_{2}$

CHRISTOPHER SKINNER

Commensurators of solvable $S$-arithmetic groups

\section{DANIEL STUDENMUND}

Gerstenhaber brackets on Hochschild cohomology of quantum symmetric algebras and their group extensions

SARAH WITHERSPOON and GUODONG ZHOU 